ARTICLE

\title{
Direct activation of the proton channel by albumin leads to human sperm capacitation and sustained release of inflammatory mediators by neutrophils
}

\author{
Ruiming Zhao ${ }^{1,6}$, Hui Dai ${ }^{1,6}$, Rodolfo J. Arias², Gerardo A. De Blas ${ }^{2,3}$, Gerardo Orta4, Martín A. Pavarotti², \\ Rong Shen ${ }^{5}$, Eduardo Perozo ${ }^{5}$, Luis S. Mayorga ${ }^{2}$, Alberto Darszon ${ }^{4} \&$ Steve A. N. Goldstein (1) ${ }^{1 凶}$
}

Human voltage-gated proton channels ( $\mathrm{hHv} 1$ ) extrude protons from cells to compensate for charge and osmotic imbalances due metabolism, normalizing intracellular $\mathrm{pH}$ and regulating protein function. Human albumin (Alb), present at various levels throughout the body, regulates oncotic pressure and transports ligands. Here, we report Alb is required to activate $\mathrm{hHv1}$ in sperm and neutrophils. Dose-response studies reveal the concentration of Alb in semen is too low to activate hHv1 in sperm whereas the higher level in uterine fluid yields proton efflux, allowing capacitation, the acrosomal reaction, and oocyte fertilization. Likewise, Alb activation of $\mathrm{hHv} 1$ in neutrophils is required to sustain production and release of reactive oxygen species during the immune respiratory burst. One Alb binds to both voltage sensor domains (VSDs) in $\mathrm{hHv} 1$, enhancing open probability and increasing proton current. A computational model of the Alb-hHv1 complex, validated by experiments, identifies two sites in Alb domain II that interact with the VSDs, suggesting an electrostatic gating modification mechanism favoring the active "up" sensor conformation. This report shows how sperm are triggered to fertilize, resolving how hHv1 opens at negative membrane potentials in sperm, and describes a role for Alb in physiology that will operate in the many tissues expressing hHv1.

\footnotetext{
${ }^{1}$ Departments of Pediatrics and Physiology \& Biophysics, Susan and Henry Samueli College of Health Sciences, University of California, Irvine, CA, USA.

2 Instituto de Histología y Embriología de Mendoza (IHEM/CONICET-UNCuyo), School of Medicine, National University of Cuyo, Mendoza, CP, Argentina. ${ }^{3}$ Laboratorio de Telediagnóstico e Investigación Traslacional (LaTIT). Área de Farmacología. Departamento de Patología, School of Medicine, National University of Cuyo, Mendoza, CP, Argentina. ${ }^{4}$ Departamento de Genética del Desarrollo y Fisiología Molecular, Instituto de Biotecnología, Universidad Nacional Autónoma de México, Morelos, México. ${ }^{5}$ Department of Biochemistry and Molecular Biology, Gordon Center for Integrative Science, University of Chicago, Chicago, IL, USA. ${ }^{6}$ These authors contributed equally: Ruiming Zhao, Hui Dai. ${ }^{{ }}$email: sgoldst2@uci.edu
} 
R egulation of intracellular $\mathrm{pH}\left(\mathrm{pH}_{\mathrm{i}}\right)$ is essential to cell biology in health and disease ${ }^{1}$ and, since its identification ${ }^{2,3}$, the human voltage-gated proton channels (hHv1) has been recognized to be both widespread and central in many of these processes $^{4}$. Recently, we used a designed peptide inhibitor (C6) of the channel to demonstrate that $\mathrm{H}^{+}$efflux via $\mathrm{hHv1}$ is required in human sperm to induce intracellular alkalization and initiate capacitation, and essential in human neutrophils to maintain cytoplasmic $\mathrm{pH}$ during the respiratory burst to allow reactive oxygen species (ROS) production ${ }^{5}$, in support of earlier proposals ${ }^{6-10}$.

hHvl channels are comprised of two identical subunits, each with four transmembrane spans (TMs) that resemble the voltage sensor domains (VSDs) in other voltage-gated ion channels (VGICs) but lack the two additional TMs that contribute to forming the ion conduction pores in those channels ${ }^{2,3}$. In hHvl, there are two $\mathrm{H}^{+}$-selective conduction pathways, one in each subunit ${ }^{11-13}$.

Albumin (Alb), is the most abundant protein in interstitial fluids where it is present at various levels $(7-30 \mathrm{mg} / \mathrm{mL})^{14}$ and human plasma (34-54 mg/mL) where it is recognized to transport hormones, metabolites and drugs, serves as a circulating antioxidant, and supports oncotic pressure ${ }^{15}$. This ubiquitous globular protein is composed of 585 amino acids and folds into three domains ${ }^{16}$.

We wondered about a physiological connection between Alb and hHvl based on its contrasting concentrations in semen (just $1 \mathrm{mg} / \mathrm{mL}, 15 \mu \mathrm{M})^{17}$ and in uterine fluid $(34 \mathrm{mg} / \mathrm{mL}, 500 \mu \mathrm{M})^{18}$ and the documented increase in reproductive success when in vitro fertilization (IVF) solutions are supplemented with $\mathrm{Alb}^{19,20}$. Furthermore, Alb is implicated in deleterious systemic inflammatory responses mediated by neutrophils after cardiopulmonary bypass and in periodontal disease ${ }^{21,22}$.

Here, we demonstrate that Alb binds directly to hHv1 to activate the channel, increasing the open probability and $\mathrm{H}^{+}$ current. In human sperm, this initiates capacitation while in human neutrophils it increases peak levels of ROS release, sustains ROS production during the respiratory burst, and stimulates release of proteases in response to immune stimulation. The stoichiometry of binding, inferred by the Hill coefficient for changes in $\mathrm{H}^{+}$currents in response to Alb dose, is confirmed to be one Alb per channel using single molecule total internal reflection fluorescent (smTIRF) microscopy. Alb is shown to bind to hHv1 on the external residues linking the third and fourth transmembrane segments that comprise the voltage sensor (S3-S4 loop) in each subunit using point mutations and chimeric channels generated between hHv1 and the proton channel from Ciona intestinalis, $\mathrm{CiHv1}^{3}$. Modeling with molecular dynamics (MD) simulation points to two binding sites formed by residues in Alb domain II (DII) for the two S3-S4 loops in each hHv1 channel. The two Alb sites are validated by the effects of mutagenesis on binding as assessed by fluorescence resonance energy transfer (FRET) microscopy and electrophysiology. The structural model of the Alb-hHv1 macromolecular complex suggests an electrostatic gating modification mechanism through which binding of Alb facilitates opening of hHvl channels by favoring the active "up" conformation of the two VSDs. The essential stimulatory role of Alb in the physiology of both sperm and neutrophils via hHv1 suggests that Alb will have as-yet unrecognized roles in the many other tissues where the channel is critical, including, the heart, the central nervous system, and cancers of the breast and gastrointestinal tract ${ }^{23,24}$.

\section{Results}

Alb acts on hHvl in human sperm to initiate capacitation. Human sperm undergo a process called capacitation in the female reproductive tract ${ }^{25}$, whereby $\mathrm{pH}_{\mathrm{i}}$ rises stimulating $\mathrm{Ca}^{2+}$ influx and mobility changes ${ }^{26}$. This process endows sperm with the capacity to undergo the exocytotic acrosomal reaction required to penetrate the zona pellucida and fertilize the oocyte ${ }^{27}$. Here, Alb was observed to activate hHv1 channels in live human sperm by recording proton currents in mature non-capacitated spermatozoa, using whole-cell patch clamp as previously described ${ }^{5}$.

The native proton channel currents were elicited by depolarizing voltage steps of $1.5 \mathrm{~s}$ from a holding potential of $-60 \mathrm{mV}$ every $10 \mathrm{~s}$ with a $\sim 30$-fold proton gradient $\left(\mathrm{pH}_{\mathrm{i}}=6.0\right.$ and $\left.\mathrm{pH}_{\mathrm{o}}=7.4\right)$. When $80 \mu \mathrm{M}$ Alb was applied to the sperm, the currents increased $\sim 3$-fold, accompanied by a $\sim 3$-fold acceleration of activation and a $\sim 1$.6-fold slowing of deactivation at $+60 \mathrm{mV}$ (Fig. 1a, left; Supplementary table 1). Applying different concentrations of Alb yielded an $\mathrm{EC}_{50}$ $=158 \pm 16 \mu \mathrm{M}$ with a Hill coefficient of $1.09 \pm 0.01$. The mean concentration of Alb $(15 \mu \mathrm{M})$ in human semen was too low to significantly activate hHvl, while the level in the uterus $(500 \mu \mathrm{M})$ increased sperm proton current $\sim 6$-fold at $+60 \mathrm{mV}$ (Fig. 1b), as did $800 \mu \mathrm{M}$ Alb in association with a half-maximal shift in the conductance-voltage $(\mathrm{G}-\mathrm{V})$ relationship $\left(\mathrm{V}_{1 / 2}\right)$ of $-32 \mathrm{mV}$ (Fig. 1b, c). In contrast, addition of a control soluble protein of similar mass, the Fab fragment of human immunoglobulin $\mathrm{G}$ (Fab), had no effect on the proton currents in sperm, whereas Alb-activated currents were suppressed by $1 \mu \mathrm{M}$ C6, indicating that they were passed by hHv1 channels (Fig. 1a, right). As $\mathrm{H}^{+}$currents depend on driving force, and a variety of proton concentrations $\left(\mathrm{pH}_{\mathrm{i}}\right.$ and $\left.\mathrm{pH}_{\mathrm{o}}\right)$ and cell types are studied in this report, the effects of Alb are collated in Supplementary table 1 .

We next focused on the effect of $\mathrm{Alb}$ on $\mathrm{pH}_{\mathrm{i}}$, as capacitation first requires $\mathrm{H}^{+}$efflux via hHv1 to alkalinize sperm ${ }^{5-7}$. The fluorescent ratiometric $\mathrm{pH}$ probe $\mathrm{BCECF}$ was used to evaluate changes in the cytosolic $\mathrm{pH}$, as previously described ${ }^{28}$. As anticipated, $75 \mu \mathrm{M}$ Alb induced a robust increase in $\mathrm{pH}_{\mathrm{i}}$ in noncapacitated human sperm when compared to a control Fab protein or after blocking hHv1 with C6 (Fig. 1d). Notably, increasing levels of Alb led to greater cytoplasmic alkalization consistent with a dose-dependent increase in $\mathrm{H}^{+}$efflux and changes in $\mathrm{pH}_{\mathrm{i}}$ were suppressed by the hHv1 channel blocker C6 (Fig. 1d, Supplementary Fig. 1a).

To confirm that Alb-induced changes in $\mathrm{pH}_{\mathrm{i}}$ increased $\mathrm{Ca}^{2+}$ influx through CatSper ${ }^{5-7}$, non-capacitated sperm incubated with Alb were exposed to progesterone, a stimulus that triggers the acrosome reaction only if sperm have been capacitated by changes in $\mathrm{pH}_{\mathrm{i}}$ and intracellular calcium levels $\left(\left[\mathrm{Ca}^{2+}\right]_{\mathrm{i}}\right)^{29,30}$. In a concentration dependent manner, Alb enhanced progesteroneinduced increases in $\left[\mathrm{Ca}^{2+}\right]_{\mathrm{i}}$, a change that was suppressed by the hHv1 blocker C6 (Fig. 1e, Supplementary Fig. 1b, c). Confirming that $\mathrm{Ca}^{2+}$ influx was mediated by CatSper, the increase in $\left[\mathrm{Ca}^{2+}\right]_{\mathrm{i}}$ was also inhibited by $1 \mu \mathrm{M}$ NNC (Supplementary Fig. 1c). Verifying that Alb did not alter dye loading to produce an artefactual change in measured $\left[\mathrm{Ca}^{2+}\right]_{\mathrm{I}}$, applying the $\mathrm{Ca}^{2+}$ ionophore ionomycin to samples after progesterone stimulation produced the same absolute rise in peak $\left[\mathrm{Ca}^{2+}\right]_{\mathrm{i}}$ in the presence and absence of $75 \mu \mathrm{M}$ Alb (Supplementary Fig. 1,d, e, f). Furthermore, Alb was shown to have no direct effect on CatSper currents in human sperm (Supplementary Fig. 2), consistent with a report that CatSper was sensitive to bovine serum albumin (BSA) indirectly 31

The final step, the exocytotic acrosomal reaction, requires prior capacitation $^{30}$ and was rarely observed $(\sim 2 \%)$ when we added progesterone to non-capacitated sperm in the absence of Alb (Fig. 1f). In contrast, when $75 \mu \mathrm{M}$ Alb was added to noncapacitated sperm $\sim 10 \%$ of the sperm underwent exocytosis, an increase that was eliminated when sperm were incubated with the hHv1 blocker C6 (Fig. 1f). Thus, activation of hHv1 by Alb is essential for sperm alkalization and CatSper potentiation (that is, capacitation) and exocytosis (that is, the acrosomal reaction $)^{30}$. 
a

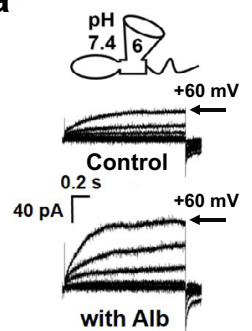

d

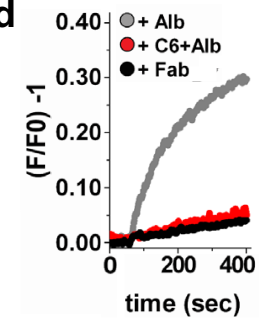

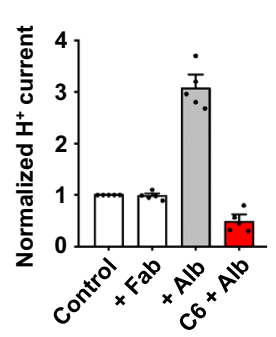

b

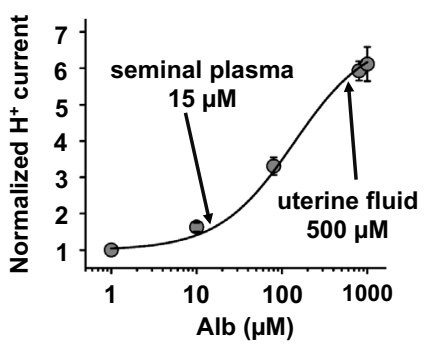

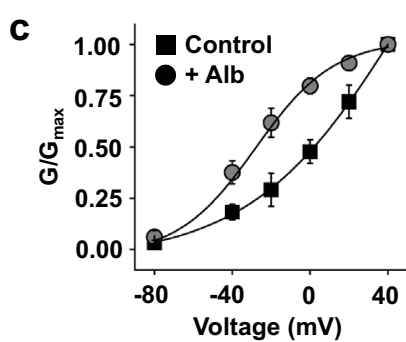

e

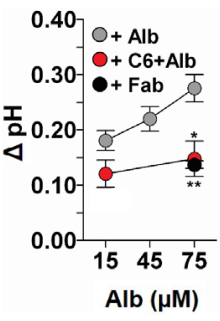

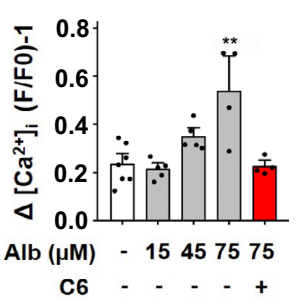

f

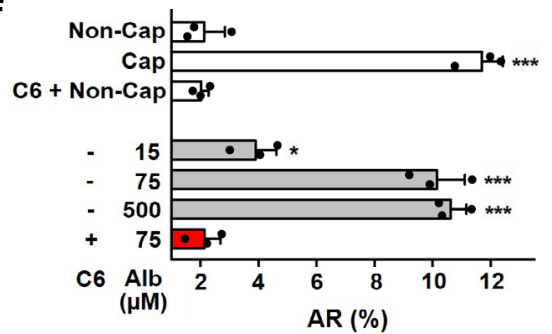

Fig. 1 Alb activates hHv1 in human sperm to initiate capacitation and allow the acrosome reaction. a Left, Proton current traces in non-capacitated human sperm in the absence (top) and presence (bottom) of $80 \mu \mathrm{M} \mathrm{Alb} \mathrm{(20} \mathrm{mV} \mathrm{steps} \mathrm{from}-60$ and $+60 \mathrm{mV}$ ). Right, hHv1 currents at the end of a test pulse to $+60 \mathrm{mV}$ with $80 \mu \mathrm{M}$ Fab (white bar), $80 \mu \mathrm{M} \mathrm{Alb}$ (gray bar), or $80 \mu \mathrm{M} \mathrm{Alb}+1 \mu \mathrm{M} \mathrm{C} 6$ (red bar). Values are normalized to mean current amplitude without Alb. C6 peptide blocked $86 \pm 3 \%$ of the Alb-activated current, $n=5$ cells. b Dose-response relationships for Alb potentiation of sperm proton current at $+60 \mathrm{mV}$. The half-maximal effective concentration $\left(E_{50}\right)$ of Alb activation estimated from the fit to Hill relationship as $158 \pm 16 \mu \mathrm{M}$ with a coefficient of $1.09 \pm 0.01, n=5$ cells. c Conductance-voltage relationships (G-V) for sperm proton currents in the absence (black squares) or presence of $800 \mu \mathrm{M}$ Alb (gray circles). Curves were fit to a Boltzmann equation, $n=4$ cells. $\mathbf{d}$ Left, Non-capacitated sperm were loaded with BCECF and changes of fluorescence intensity measured. $75 \mu \mathrm{M} \mathrm{Alb}$ (gray trace) increased $\mathrm{pH}_{\mathrm{i}}$ and the increase was inhibited by $20 \mu \mathrm{M} \mathrm{C6}$ (red trace). A $75 \mu \mathrm{M} \mathrm{Fab}$ alone had no effect (black trace). Right, BCECF signals were converted to $\Delta \mathrm{pH}$ as described in Methods. Alb-induced cytoplasmic alkalization was concentration dependent (gray circles). The Alb-triggered $\mathrm{pH}_{\mathrm{i}}$ increase was inhibited by $\mathrm{C} 6$ (red circles, ${ }^{\star} P=0.03$; one way ANOVA, Dunnett Test); Fab did not increase the $\mathrm{pH}_{\mathrm{i}}$ (black circle, ${ }^{\star \star} P=0.005$; one way ANOVA, Dunnett Test), $n=3-13$ independent experiments (Supplementary Fig. 1a). e Non-capacitated sperm were loaded with Fluo-3 and changes in fluorescence measured. Alb potentiates the increase of $\left[\mathrm{Ca}^{2+}\right]_{i}$ induced by progesterone $(15 \mu \mathrm{M})$ in a concentration dependent manner (gray bars). The $\left[\mathrm{Ca}^{2+}\right]_{i}$ increase potentiated by $75 \mu \mathrm{M}$ Alb was $2.3-$ fold ( ${ }^{\star \star} P=0.002$; one way ANOVA, Dunnett Test) and was fully suppressed by $20 \mu \mathrm{M} \mathrm{C} 6$ peptide (red bar), $n=4-7$ independent experiments (Supplementary Fig. 1b, c). f Acrosomal exocytosis induced by $15 \mu \mathrm{M}$ progesterone. Upon progesterone stimulation control capacitated sperm underwent the acrosome reaction (Cap, white bar, ${ }^{\star \star \star} P=0.0001$; one way ANOVA, Dunnett Test), whereas non-capacitated sperm did not (Non-Cap, white bar). Incubation of C 6 peptide (20 $\mu$ M) with non-capacitated sperm had no effect ( 66 + Non-Cap, white bar). In a concentration dependent manner, incubation with Alb increased the progesterone-induced acrosome reaction (gray bars, ${ }^{\star} P=0.04$; one way ANOVA, Dunnett Test) in non-capacitated sperm; addition of C6 (20 $\mu \mathrm{M}$, red bar) fully inhibited exocytosis stimulated by $75 \mu \mathrm{M}$ Alb, $n=3$ independent experiments. Values are mean \pm SEM. Source data are provided in the Source Data file.

Alb stimulates hHvl in neutrophils to augment ROS production and elastase release. Human neutrophils undergo a respiratory burst to produce ROS as a principal effector mechanism to kill bacteria ${ }^{32}$. During the respiratory burst, the NADPH oxidase 2 (NOX2) transfers electrons across the membrane, resulting in membrane depolarization and cytoplasmic acidification that suppresses ROS production ${ }^{33}$. To sustain NOX2 activity and ROS production, $\mathrm{H}^{+}$efflux is required to maintain physiological $\mathrm{pH}_{\mathrm{i}}$ and membrane potential ${ }^{23,34}$.

Here, activation of hHvl in neutrophils by Alb was demonstrated first by recording native proton currents in freshlyisolated cells from human peripheral blood by whole-cell patch clamp. Application of $450 \mu \mathrm{M}$ Alb led to a 2.5 -fold increase in native proton currents at $+60 \mathrm{mV}$, with a $\sim 6$-fold acceleration of activation and a $\sim 1.5$-fold slowing of deactivation, offering an estimated equilibrium affinity $\left(K_{d}\right)$ of $112 \pm 9 \mu \mathrm{M}$ (Fig. 2a, left) and a shift of $-35 \mathrm{mV}$ in the G-V relationship (Supplementary Fig. 3a and Supplementary Table 1). As observed with sperm, Fab application had no effect on the current and Alb-activated proton currents were suppressed by $20 \mu \mathrm{M} \mathrm{C} 6$, demonstrating their passage by hHv1 (Fig. 2a, right).

To assess ROS release from the neutrophils, we used a luminolamplified chemiluminescence assay for superoxide anion $\left(\mathrm{O}_{2}{ }^{--}\right)$ measurement ${ }^{35}$. Formylated bacterial peptides like fMLF stimulate the production of ROS by neutrophils by binding to G-protein coupled receptors ${ }^{36}$ and triggering intracellular pathways that induce NOX2 to produce $\mathrm{O}_{2} \cdot{ }^{\cdot-4},{ }^{37}$. Here, we observed that

$1 \mu \mathrm{M}$ fMLF stimulated a transient rise and decay in ROS release from neutrophils as expected, whereas the additional presence of $450 \mu \mathrm{M}$ Alb led to an increase in the peak magnitude of ROS release. More significantly, Alb allowed for sustained ROS production that was still elevated at $60 \mathrm{~min}$, long after release had returned to baseline in the absence of Alb (Fig. 2b, left).

ROS release potentiated by Alb was concentration-dependent across the physiological range, becoming apparent at $15 \mu \mathrm{M}$ and maintained at a $\sim 7$-fold increased rate of release after $60 \mathrm{~min}$ with $450 \mu \mathrm{M}$ (Fig. 2b, right). As expected, neither augmentation of peak ROS release, nor sustained ROS production, was observed with $450 \mu \mathrm{M} \mathrm{Fab}$, and potentiation was fully inhibited by $20 \mu \mathrm{M}$ C6 (Fig. 2b, right). In the absence of immune stimulation by fMLF, Alb induced no increase in ROS release above low basal levels, consistent with a role as a potentiator and not the primary stimulus (Supplementary Fig. 3b). Furthermore, Alb potentiation of total ROS production was stimulated with as little as $10 \mathrm{nM}$ fMLF ( 1.8-fold) and rose to $\sim 5$-fold with $10 \mu \mathrm{M}$ fMLF without a shift in the fMLF dose-response curve (Fig. 2c, Supplementary Fig. 3c, d). 
a

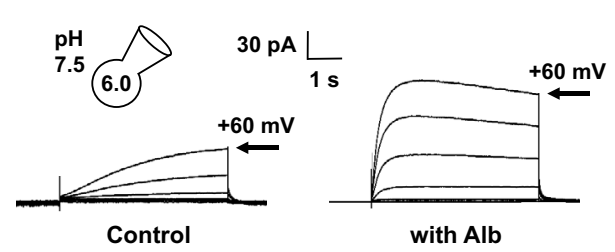

b
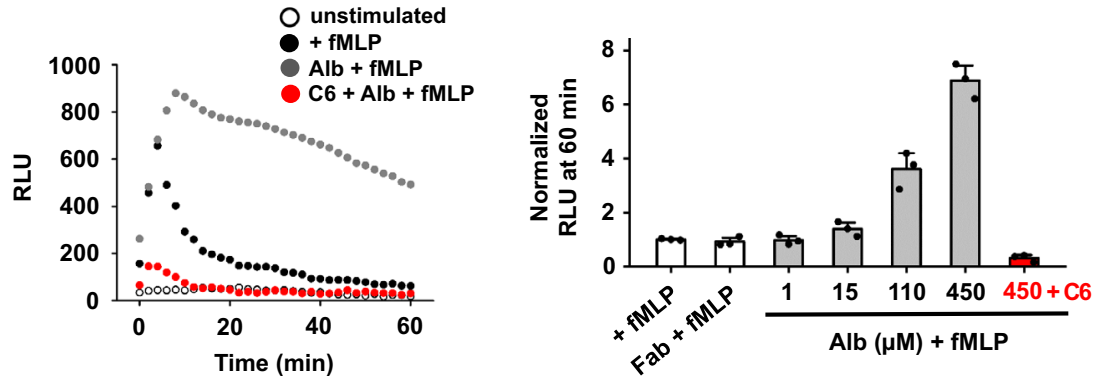

C
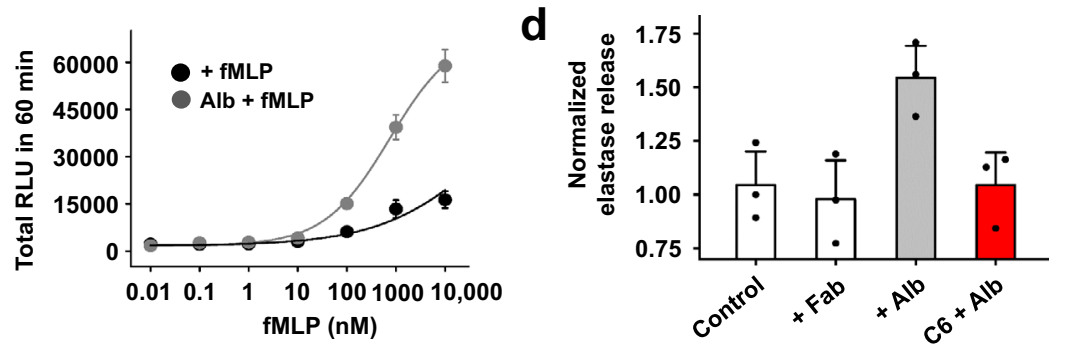

Fig. 2 Alb activates hHv1 in human neutrophils to increase ROS production and elastase release. a Proton currents passed by hHv1 in human neutrophils studied in the absence (left) and presence (middle) of $450 \mu \mathrm{M} \mathrm{Alb}$ (steps from -60 and $+60 \mathrm{mV}$ in $20 \mathrm{mV}$ increments). Fitting activation and deactivation of currents at $+60 \mathrm{mV}$ offered time constants (Supplementary Table 1). Right, hHv1 currents measured at the peak of a test pulse to $+60 \mathrm{mV}$ after exposing neutrophils to $450 \mu \mathrm{M} \mathrm{Fab}$ (white bar), $450 \mu \mathrm{M}$ Alb (gray bar), or $450 \mu \mathrm{M} \mathrm{Alb}+20 \mu \mathrm{M} \mathrm{C6}$ (red bar). Values are normalized to mean current amplitude in the absence of Alb. C6 peptide blocked $87 \pm 2 \%$ of of the current activated by Alb, $n=3$ cells. b Left, effect of fMLF alone or in combination with Alb on ROS production by human neutrophils. Human neutrophils $\left(2 \times 10^{5}\right.$ cells) were incubated without or with $450 \mu \mathrm{M}$ Alb, then stimulated with $1 \mu \mathrm{M}$ fMLF. ROS was measured using luminol (relative light units, RLU). ROS production from unstimulated neutrophils is negligible (white circles). Alb (gray circles) enhanced and sustained the fMLF-stimulated ROS production compared to neutrophils stimulated with fMLF alone (black circles). Incubation with $20 \mu \mathrm{M}$ C6 (red circles) inhibited ROS production potentiated by $450 \mu \mathrm{M} \mathrm{Alb.} \mathrm{Right,} \mathrm{ROS} \mathrm{produced} 60$ min after fMLF stimulation. Incubation with Alb increased ROS production by human neutrophils (gray bars); addition of C6 (20 $\mu \mathrm{M}$, red bar) inhibited the ROS production potentiated by $450 \mu \mathrm{M}$ Alb. $450 \mu \mathrm{M}$ Fab had no effect on fMLF-stimulated ROS production (white bar). Values are normalized to mean ROS production by neutrophils stimulated with $\mathrm{fMLF}$ alone, $n=3$ independent experiments. c Human neutrophils ( $2 \times 10^{5}$ cells) were incubated without (black circles) or with $450 \mu \mathrm{M}$ Alb (gray circles) and then stimulated with increasing concentrations of fMLF. ROS were measured as described in (b). Some error bars are smaller than symbols, $n=3$ independent experiments. d Human neutrophils ( $6 \times 10^{5}$ cells) were incubated with Alb and then stimulated with $1 \mu \mathrm{M}$ fMLF. Total elastase release at each test condition was measured spectrophotometrically. The values are normalized to elastase release stimulated with $\mathrm{fMLF}$ alone (Control, white bar). A $450 \mu \mathrm{M}$ Alb increased the elastase release by neutrophils (gray), and the increase was inhibited by $20 \mu \mathrm{M} \mathrm{C6}$ (red) whereas $450 \mu \mathrm{M}$ Fab had no effect on $\mathrm{fMLF}$-stimulated elastase release (white bar), $n=3$ independent experiments. Values are mean \pm SEM. Source data are provided in the Source Data file.

During the inflammatory response, neutrophils also release antimicrobial proteases that cleave bacterial virulence factors, such as elastase ${ }^{38}$. Thus, fMLF-induced neutrophil degranulation can be quantified by measurement of elastase release ${ }^{21}$. Fig. 3d shows that Alb augmented fMLF-stimulated elastase release $\sim 1.5$ fold whereas the same amount of Fab had no effect and, demonstrating dependence on $\mathrm{H}^{+}$efflux via hHvl, augmentation was suppressed by $20 \mu \mathrm{M}$ C6 (Fig. 2d).

hHvl shows an "enhanced gating mode" during the neutrophil respiratory burst increasing the likelihood of channel opening that is reported to reflect protein kinase $\mathrm{C}(\mathrm{PKC})$ phosphorylation of Thr29 at $\mathrm{N}$-terminus of $\mathrm{hHv}^{39}$. Indicating that Alb-activation was not mediated by this mechanism, we observed that hHv1T29A channels, which are not subject to PKC phosphorylation, were activated by Alb like WT channels (Supplementary Fig. 4).
Alb also activates cloned hHv1 by changing the voltagedependence of gating. To explore the mechanism of Alb activation, hHv1 channels were expressed in HEK293T cells and studied by whole-cell patch clamp with a 10 -fold proton gradient $\left(\mathrm{pH}_{\mathrm{i}}=6.5\right.$ and $\left.\mathrm{pH}_{\mathrm{o}}=7.5\right)$, as previously described ${ }^{5}$. In the absence of Alb, hHvl currents activated slowly in response to depolarization and showed fast deactivation (Fig. 3a, left). When $75 \mu \mathrm{M}$ Alb was added to the bath solution, hHvl currents increased $\sim 5$-fold due to a $\sim 8$-fold acceleration of activation, a $\sim 3$-fold slowing of deactivation at $0 \mathrm{mV}$, and a shift in the $\mathrm{V}_{1 / 2}$ of $-23 \mathrm{mV}$ (Fig. 3b and Supplementary Table 1).

Alb stimulation of hHvl currents was reversible and concentrationdependent with association and dissociation constants of $k_{\text {on }}=1.3 \times$ $10^{3} \pm 0.1 \times 10^{3} \mathrm{M}^{-1} \mathrm{~s}^{-1}$ and $k_{\text {off }}=0.084 \pm 0.009 \mathrm{~s}^{-1}$ determined by single-exponential fits to the time courses for activation and 
a
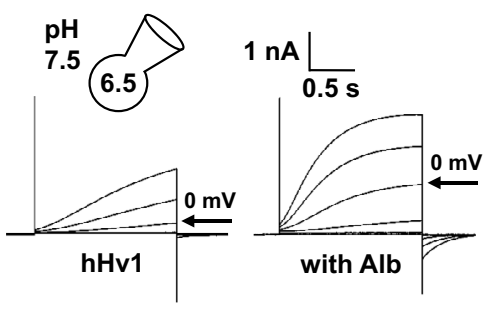

C

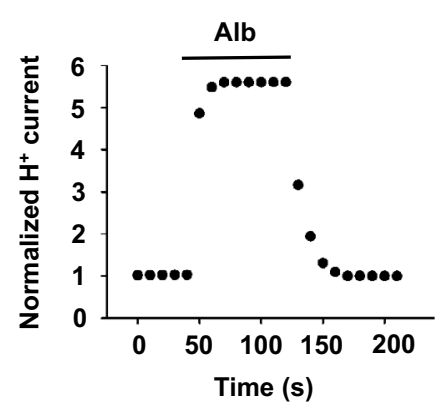

d b
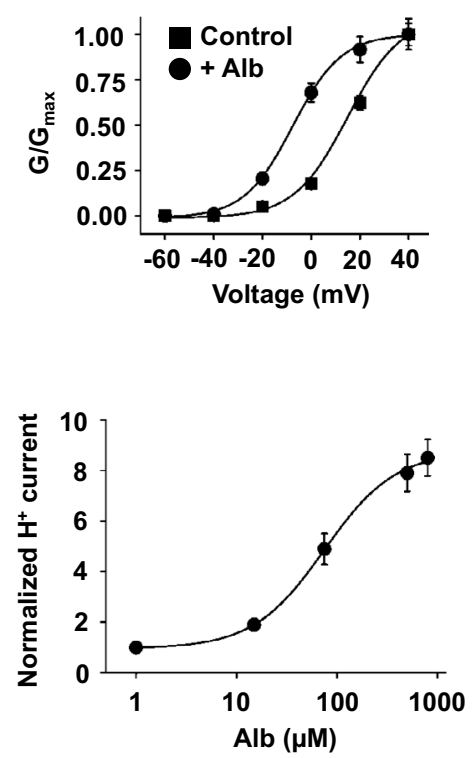

$0.5 \mathrm{nA}$

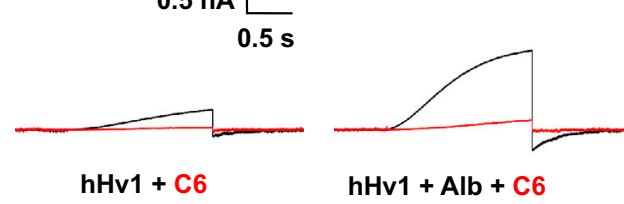

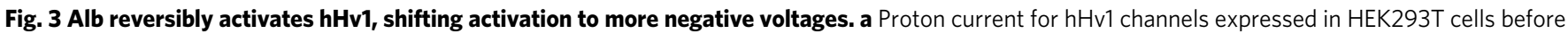
(left), and in the presence of $75 \mu \mathrm{M}$ Alb (right), with steps of $20 \mathrm{mV}$ from $-60 \mathrm{mV}$ to $+40 \mathrm{mV}$. The current measured at the end of depolarization was used to determine the extent of activation. Fitting the activation and deactivation of proton currents at $0 \mathrm{mV}$ to a single exponential function gave time constants $\tau_{\text {act }}$ of $2619.8 \pm 222.3 \mathrm{~ms}$ and $332.3 \pm 30.6 \mathrm{~ms}, \tau_{\text {tail }}$ of $86.9 \pm 8.4 \mathrm{~ms}$ and $235.0 \pm 29.7$ ms without and with Alb, respectively. b G-V for hHv1 in the absence (black squares) or presence of $75 \mu \mathrm{M}$ Alb (black circles). hHv1 channels showed a $-23 \pm 3 \mathrm{mV}$ shift after exposure to $75 \mu \mathrm{M} \mathrm{Alb}$ from $15 \pm 2 \mathrm{mV}$ to $-8 \pm 1 \mathrm{mV}$. Curves fit to a Boltzmann equation, $n=6$ cells. c Time course for activation and deactivation of hHv1 currents on acute application (bar) and washout of $75 \mu \mathrm{M} \mathrm{Alb}$. Currents were recorded at $0 \mathrm{mV}$. Values are normalized to the control current before the application of Alb. d Dose-response relationships for Alb activation of $\mathrm{hHv} 1$ at $0 \mathrm{mV}$. The $\mathrm{EC}_{50}$ was estimated from a fit to the Hill relationship to be $74.8 \pm 8.7 \mu \mathrm{M}$ with a coefficient of $1.16 \pm$ 0.11. Values are normalized to mean proton currents amplitude in the absence of Alb, $n=6-8$ cells. e Cartoon showing two C 6 peptides binding to an intact dimeric hHv1 channel. f C6 peptide ( $20 \mu \mathrm{M}$, red trace) was applied to cells after control pulses (black trace) without Alb (left) or after pre-activation with $75 \mu \mathrm{M}$ Alb (right). C6 inhibited $\sim 92 \%$ of hHv1 proton current (left), which is similar to the extent of inhibition ( 89\%) with Alb pre-activation (right). Values are mean \pm SEM. Some error bars are smaller than symbols. Source data are provided in the Source Data file.

deactivation, respectively (Fig. 3c). Applying different concentrations of Alb yielded an $\mathrm{EC}_{50}=74.8 \pm 8.7 \mu \mathrm{M}$ with a Hill coefficient of $1.16 \pm$ 0.11 , consistent with the binding of one Alb per hHv1 dimer (Fig. 3d). Moving sperm from the concentration of Alb in human semen (15 $\mu \mathrm{M})$ to that in the uterus $(500 \mu \mathrm{M})$, increased hHvl currents $\sim 8$-fold at $0 \mathrm{mV}$, shifting the $\mathrm{V}_{1 / 2}$ by $-45 \mathrm{mV}$ (Fig. 3d and Supplementary Fig. 5a, b).

The designed peptide C6 inhibits hHv1 by binding to the S3-S4 loops with positive cooperativity, one peptide on each loop (Fig. 3e), thereby biasing the voltage sensors to the down conformation that favors channel closure ${ }^{5}$. As a result, more positive voltages are required to open the channel pores, opening is slowed, and closing is faster; the reverse of the effects of Alb on the channel. Confirming that Alb-induced currents were due to $\mathrm{H}^{+}$permeation through hHv1, $20 \mu \mathrm{M}$ C6 suppressed nearly all the proton current (Fig. 3f).

This effect of Alb showed specificity and required that the protein was intact, as neither Fab nor Alb subjected to Proteinase $\mathrm{K}$ digestion activated $\mathrm{hHvl}$ at concentrations up to $800 \mu \mathrm{M}$ (Supplementary Fig. 6). Specificity of Alb for hHv1 was further demonstrated by failure of Alb to activate hKv1.3, hKv2.1, hKv1.5, hI $\mathrm{Ks}_{\text {s }}$ (hKv7.1 + hKCNE1), and hNav1.5 (Supplementary Fig. 7) although this represents only a sampling of the many
VGICs. Alb activated hHv1 only from the extracellular solution, showing no effect from the cytosol when introduced via the pipette solution ( $75 \mu \mathrm{M}, n=3$ cells), and was observed also to activate hHv1 expressed in Xenopus oocytes (Supplementary Fig. 8).

A recent report indicates that a protease-digested form of hHv1 (Hv1Sper) with 68 residues removed from the N-terminus represents as much as half the channel protein isolated from human sperm when visualized by western blotting ${ }^{40}$. We constructed the foreshortened channel by genetic deletion of the residues, expressed it in HEK293T cells and observed the approximately $-30 \mathrm{mV}$ shift in the G-V relationship compared to WT hHv1 reported by Berger and colleagues ${ }^{40}$. Indicating that the truncated channels are also sensitive to Alb regulation, we observed application of $500 \mu \mathrm{M}$ Alb to accelerate activation, slow deactivation, increase current magnitude, and to shift the G-V curve by -15 $\mathrm{mV}$ (Supplementary Table 1 and Supplementary Fig. 9).

Because Human Tubal Fluid Medium (HTF, pH 7.2) is routinely used for human $\mathrm{IVF}^{41}$ to mimic the native environment, we sought to confirm that the effect of Alb on hHv1 channels was apparent in this solution using a pipette solution at $\mathrm{pH} 6.7$ as estimated for sperm in the human uterus ${ }^{42}$. Indeed, under these conditions, $500 \mu \mathrm{M}$ Alb increased hHvl current $\sim 10$ - 
fold at $0 \mathrm{mV}$, shifting the $\mathrm{V}_{1 / 2}$ for conduction by $-40 \mathrm{mV}$ (Supplementary table 1 and Supplementary Fig. 10). As a result, the voltage at which hHv1 channels start to open $\left(\mathrm{V}_{\text {threshold }}\right)$ shifted from $\sim 0 \mathrm{mV}$ to $-30 \mathrm{mV}$ with Alb (Supplementary Fig. 10). These values are comparable to those observed for native proton currents in sperm where Alb produced an approximately -32 $\mathrm{mV}$ hyperpolarization in the $\mathrm{V}_{1 / 2}$ and shifted the $\mathrm{V}_{\text {threshold }} \sim-30$ $\mathrm{mV}$ (Fig. 1c).

Alb binds to the external S3-S4 loop of hHv1. We looked for Alb binding sites on the extracellular portions of hHvl, that is, the residues linking the four membrane-spanning segments, the S1-S2 loop and the S3-S4 loop (Fig. 3e). First, we exchanged the loops in hHv1 with those in CiHvl, a homologous proton channel insensitive to $75 \mu \mathrm{M}$ Alb, observing when the S1-S2 loop and/or the S3-S4 loops were switched between CiHv1 and hHv1, the channels were functional (Fig. $4 \mathrm{a}, \mathrm{b}$ ).

CiHv1 channels carrying both the S1-S2 and S3-S4 loops of hHv1 ( $\left.h_{\mathrm{S} 1-\mathrm{S} 2, \mathrm{~s} 3-\mathrm{S} 4} \mathrm{CiHv} 1\right)$ were endowed with sensitivity to Alb, whereas transplanting only the S1-S2 loop from hHv1 into CiHv1 $\left(\mathrm{h}_{\mathrm{S} 1-\mathrm{S} 2} \mathrm{CiHv1}\right.$ ) was insufficient to confer Alb-activation (Fig. $4 \mathrm{a}$, b). In contrast, transferring only the S3-S4 loop from hHv1 into $\mathrm{CiHv1}$ to create $\mathrm{h}_{\mathrm{S} 3-\mathrm{S} 4} \mathrm{CiHv} 1$ conferred Alb sensitivity $\left(K_{d} \sim 63\right.$ $\mu \mathrm{M})$ at levels similar to that observed with wild type (WT) hHv1 channels (Supplementary Table 1). hHv1 carrying the S3-S4 loop of CiHv1 ( $\mathrm{Ci}_{\mathrm{S} 3-\mathrm{S} 4} \mathrm{hHv1}$ ) became insensitive to Alb (Fig. $4 \mathrm{a}, \mathrm{b}$ ), supporting the conclusion that the hHv1 S3-S4 loop was the major binding epitope for Alb. Moreover, transplanting the S3-S4 loop of hHv1 into the hKv2.1 potassium channel conferred weak potentiation by Alb (Supplementary Fig. 11), supporting the notion that the mechanism of Alb action was via the voltage sensor loop.

To explore the role of the hHv1 S3-S4 loop in Alb binding, we carried out a mutational scan where loop residues I183 to L204 were changed individually to Cys and the response of the mutant channels to Alb was studied (Fig. 4c). Two mutations, hHv1H193C and hHv1-L200C, fully eliminated Alb activation, suggesting that these two residues may mediate direct interaction with Alb. Three other changes (hHv1-E192C, hHv1-E196C, and hHv1-G199C) enhanced Alb activation. Studying these sites with further mutations yielded hHv1-G199L, a proton channel with a $\sim 22$-fold improvement in the $\mathrm{Alb}^{\mathrm{EC}} \mathrm{E}_{50}(3.4 \pm 0.5 \mu \mathrm{M})$ compared to WT hHvl and no change in the G-V relationship (Fig. $4 \mathrm{~d}$ and Supplementary Table 1). Furthermore, fitting the dose-response curve of Alb on the channel yielded a Hill coefficient of $0.93 \pm$ 0.10 like WT channels, consistent with binding of single Alb. The G199L mutant accelerated Alb association $\sim 5$-fold $\left(k_{\text {on }}=6.9 \times\right.$ $\left.10^{3} \pm 0.7 \times 10^{3} \mathrm{M}^{-1} \mathrm{~s}^{-1}\right)$ and slowed dissociation $\sim 4$-fold $\left(k_{\mathrm{off}}=\right.$ $\left.0.022 \pm 0.003 \mathrm{~s}^{-1}\right)$.

One Alb binds to one dimeric hHvl channel. The increased affinity of Alb for hHv1-G199L channels allowed a direct study of binding using smTIRF and live cells (Methods). Alb was labeled with carboxytetramethylrhodamine (TAMRA) on its N-terminus to yield TAMRA-Alb and the channel was tagged on its Cterminus with a teal fluorescent protein to produce hHv1-G199LTFP (Fig. 4e). Incubation of TAMRA-Alb with HEK293T cells expressing hHv1-G199L-TFP produced doubly-labeled particles containing both fluorescent tags at the cell surface (Fig. $4 \mathrm{f}$, left). Cells subjected to intense illumination were used to count the number of Hv1-G199L-TFP subunits and TAMRA-Alb molecules by single molecule fluorophore bleaching, as previously described 5 .

Control experiments with hHv1-G199L-TFP subunits showed two stepwise decreases in fluorescence in $89 \%$ of particles (Fig. $4 \mathrm{f}$, $\mathrm{g}$ and Supplementary Table 2), as expected from hHv1 dimeric assembly. We report few, if any, monomer channels on the cell surface (Supplementary Table 2) ${ }^{43}$, as the observed singleton events were consistent with prebleaching and missed events based on the bandwidth of the smTIRF system ${ }^{5}$.

When $3 \mu \mathrm{M}$ TAMRA-Alb was applied to cells expressing hHv1-G199L-TFP, smTIRF microscopy yielded a mean Manders' coefficient for colocalization (MCC) of $0.50 \pm 0.06$, suggesting that roughly half the channels were associated with TAMRA-Alb (Supplementary Table 3). Of the hHv1-G199L-TFP particles directly confirmed to be intact dimeric channels (that is, showing two TFP bleaching steps) and to be colocalized with TAMRAAlb, 95\% were observed to be associated with one Alb, and 5\% were visualized with two (Fig. 4f, g and Supplementary Table 3). Binding of one Alb per dimeric hHvl channel is reasonable given its dimensions estimated from $3 \mathrm{D}$ structures $\left(30 \times 80 \times 80 \AA^{3}\right.$, PDB $1 \mathrm{BM} 0)^{16}$, far exceeding the exposed surface of the dimeric hHv1 channel $\left(\sim 40 \times 40 \AA^{2}\right)^{43}$.

As an independent test of the 1 Alb to 1 dimeric hHvl channel stoichiometry, we studied monomeric hHvl $(\Delta \mathrm{hHv})$, engineered by shortening the $\mathrm{N}$-terminus and removing the $\mathrm{C}$-terminus coiled-coil domain, as described ${ }^{5}$. Supplementary Figure 12 shows that while $\Delta \mathrm{hHv} 1$ channels were operational, they did not respond to $75 \mu \mathrm{M}$ Alb. We suspect our observation that high Alb concentrations were able to activate $\Delta \mathrm{hHvl}$ with an $\mathrm{EC}_{50}=$ $1,904 \pm 155 \mu \mathrm{M}$ and a Hill coefficient of $1.05 \pm 0.06$ (Supplementary Fig. 12b), reflects the response of dimeric channels that form as a minor population with these truncated subunits ${ }^{43}$.

Alb DII is the principal domain for binding to hHvl. To facilitate characterization of the Alb-hHv1 interaction by mutational screening, we constructed a gene allowing expression of Alb variants on the extracellular surface of cells via a membrane tether. The construct was comprised by the nucleotides encoding Alb (or Alb variants) in-frame with the code for the transmembrane helix of the platelet-derived growth factor receptor carrying the fluorescent protein mVenus (VFP) on its intracellular Cterminus to produce T-Alb-VFP ("Methods") ${ }^{44}$. This permitted study of the interaction of T-Alb-VFP constructs and hHvl tagged with teal fluorescent protein on its C-terminus (hHv1TFP) in live cells (Fig. 5a) using FRET microscopy ${ }^{45}$.

When T-Alb-VFP was expressed with hHv1-TFP, it potentiated the channel to a similar extent as soluble Alb. T-Alb-VFP increased current density at $0 \mathrm{mV} \sim 3$-fold, speeding the kinetics of activation $\sim 4$-fold, slowing deactivation $\sim 2$-fold, and shifting $\mathrm{V}_{1 / 2}$ by $-12 \mathrm{mV}$, values consistent with an effective concentration of free Alb in solution of $\sim 35 \mu \mathrm{M}$ (Fig. 5b-d). Two control tethered constructs, one with $\mathrm{C} 6$ peptide (T-C6-VFP) and another with no Alb insert (T-NO-VFP), further validated the approach, producing full inhibition and no potentiation, respectively (Fig. 5b, c). This data is consistent with our prior use of other tethered toxins and channels ${ }^{46}$.

The physical interaction of T-Alb-VFP with hHv1-TFP at the membrane surface of HEK293T cells was measured using the photobleaching rate of the TFP donor (Fig. 5e). hHv1-TFP alone, and hHv1-TFP expressed with T-NO-VFP, showed a mean time constant $(\tau)$ for photobleaching of $24 \pm 4 \mathrm{~s}$ and $27 \pm 5 \mathrm{~s}$, respectively (Fig. 5f, g). Demonstrating direct interaction of hHv1-TFP and T-Alb-VFP, the $\tau$ for hHv1-TFP increased to $54 \pm 6 \mathrm{~s}$, on expression with T-Alb-VFP, and to $78 \pm 8 \mathrm{~s}$ on expression with T-C6-VFP (Fig. 5f, g). Consistent with the failure of Alb to activate CiHv1, no FRET was observed between CiHv1TFP and T-Alb-VFP (Fig. 5g).

Alb consists of three homologous domains: DI (residues D1 to R197); DII (residues L198 to Q385), and DIII (residues N386 to 
a

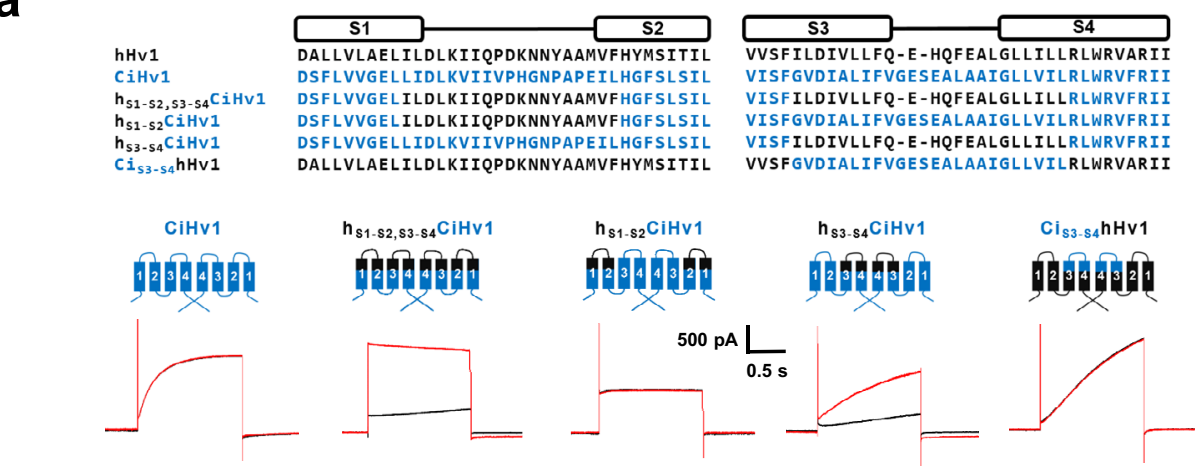

b

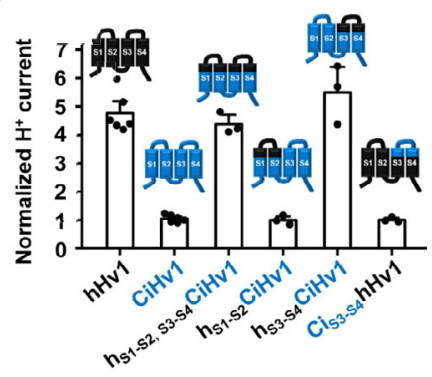

C

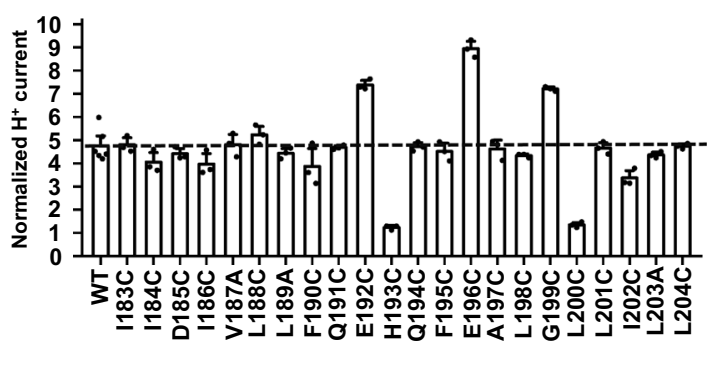

d

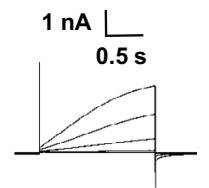

hHv1-G199L

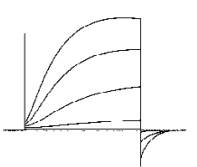

with $1 \mu \mathrm{M}$ Alb

f

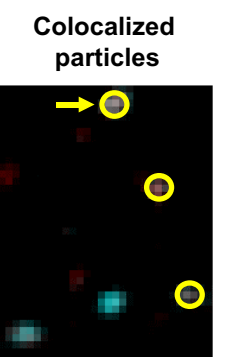

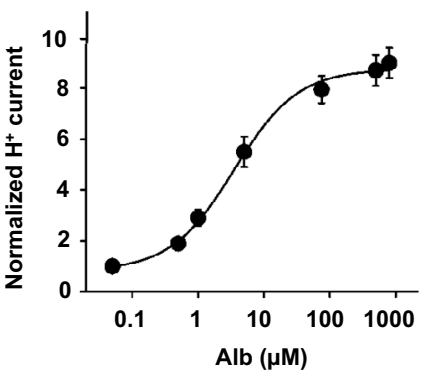

e

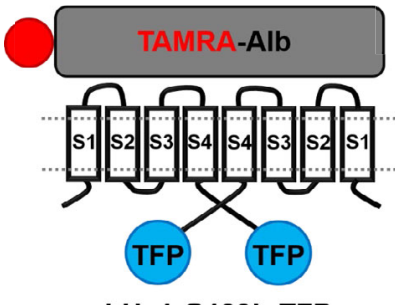

hHv1-G199L-TFP g

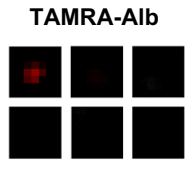
hHv1-G199L-TFP

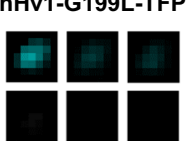

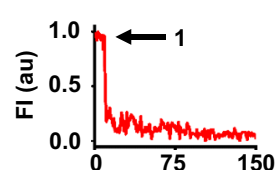

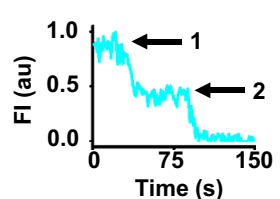

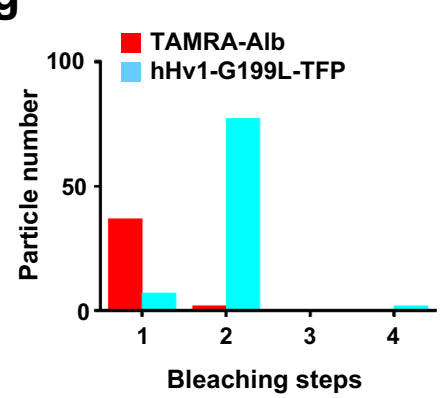

L585) 15,47 . We investigated how each domain contributed to hHv1 binding by developing six tethered Alb domain variants, TDI-VFP; T-DII-VFP; T-DIII-VFP; T-DI-DII-VFP; T-DII-DIIIVFP; and T-DI-DIII-VFP (Methods). When the interaction between tethered Alb domains and hHvl channels on the cell surface were measured by FRET, the variant consisting Alb DII alone (T-DII-VFP) interacted with hHv1-TFP like WT Alb (TAlb-VFP) (Fig. 5h). In contrast, DI alone (T-DI-VFP) appeared to interact weakly with hHvl-TFP, and T-DIII-VFP showed no evidence for a role in binding to the channel (Fig. 5h). Supporting the notion that DIII was redundant, the DI-DII variant (T-DIDII-VFP) showed an even stronger interaction than WT Alb, while T-DII-DIII-VFP showed less FRET with hHv1-TFP than DII alone (Fig. 5h). Corroborating an essential role for DII, the variant lacking that domain (T-DI-DIII-VFP) displayed greatly reduced FRET with hHv1-TFP (Fig. 5h).

In the absence of high resolution information for the Alb-hHv1 complex, we performed an in silico docking analysis (HPEPDOCK) ${ }^{48}$ using the crystal structure of Alb (PDB 1BM0) ${ }^{16}$ and the 11-residue binding epitope in S3-S4 loop of hHv1 (F190 to L200). Initial docking predicted two sites in Alb, separated by $\sim 27 \AA$, as putative hot spots for interaction (Supplementary Fig. 13a, c). This distance is comparable to that between hHv1-H193 in the two hHvl channel subunits $(\sim 26 \AA)$ in the structural model of hHvl based on electron 
Fig. 4 Alb binds to S3-S4 loop of hHv1 channels. hHv1, CiHv1, hHv1-G199L and chimeric channels were expressed in HEK293T cells and studied by wholecell patch clamp. a Top, sequence alignments of the S1-S2 and S3-S4 motifs of hHv1 (black), CiHv1 (blue) and loop-transplant chimeras. Bottom, proton current traces at $0 \mathrm{mV}$ for channels in the absence (black trace) or presence of $75 \mu \mathrm{M}$ Alb (red trace). b Activation by Alb ( $75 \mu \mathrm{M}$ ) of WT and chimeric channels, current at the end of a test pulse to $0 \mathrm{mV}$ is plotted. Values are normalized to current amplitude before Alb application. The cartoons represent the topology of a single subunit with hHv1 segments in black and CiHv1 segments in blue, $n=3-6$ cells. c Mutagenesis scan of the S3-S4 loop of hHv1 (Cys substitution except for V187A, L189A, and L203A) showing changes with $75 \mu \mathrm{M} \mathrm{Alb}$ at $0 \mathrm{mV}$; numbering corresponds to the amino acid sequence of hHv1. Values normalized to the current amplitude before Alb application, $n=3-6$ cells. $\mathbf{d}$ Left, proton current traces for hHv1-G199L channels in the absence (up) and presence of $1 \mu \mathrm{M}$ Alb (bottom) with steps of $20 \mathrm{mV}$ from $-60 \mathrm{mV}$ to $+40 \mathrm{mV}$. Right, Alb dose-response relationship for hHv1-G199L provides an $\mathrm{EC}_{50}$ for Alb activation of $3.4 \pm 0.5 \mu \mathrm{M}$ estimated by a fit to the Hill relationship with a coefficient of $0.93 \pm 0.10$. Values are normalized to mean proton current amplitude in the absence of Alb. Some error bars are smaller than symbols, $n=3-8$ cells. e Cartoon of one TAMRA-Alb molecule binding to the two subunits in a hHv1-G199L-TFP channel. $\mathbf{f}$ Left, incubation of TAMRA-Alb (red) with hHv1-G199L-TFP channels (teal) produces single colocalized particles (white) with both TAMRA and TFP fluorescence at the surface of cells (yellow circles). Middle, montage of photobleaching time course of a single fluorescent particle (arrow in left panel) during continuous excitation to bleach the fluorophores (every fifth frame is shown). Right, time courses for simultaneous photobleaching of both fluorophores in the particle, showing one stepwise change in fluorescence intensity for TAMRA-Alb and two for hHv1-G199L-TFP (arrows). g Histogram of photobleaching steps for hHv1-G199L-TFP (teal bars) and TAMRA-Alb (red bars) on simultaneous photobleaching. $89 \%$ of studied particles with hHv1-TFP were bleached in 2 steps. Data analyzed by the approach of Hines ${ }^{67}$ estimates two hHv1-G199L subunits in surface particles with a confidence of $>0.999$ (Supplementary Table 2). Among all particles containing both fluorescent colors $95 \%$ had one TAMRA-Alb bleaching step at $3 \mu$ M TAMRA-Alb (Supplementary Table 3). Values are mean \pm SEM. Source data are provided in the Source Data file.

paramagnetic resonance (EPR) spectroscopy ${ }^{43}$. Consistent with the loss-of-function mutation that fully-eliminated Alb activation (Fig. 4c), hHv1-H193 appears to be a key component of the Alb "receptor" in the S3-S4 loop. Two low energy poses from in silico docking exhibited interaction between hHv1-H193 and Alb-E188, Alb-H288, Alb-E292 in Site 1 (Supplementary Fig. 13b). Three low energy poses exhibited interaction between hHv1-H193 and AlbY334, Alb-R337, Alb-H338, Alb-D340, Alb-F374, Alb-V381 in Site 2 (Supplementary Fig. 13d). These predicted interacting residues are in Alb DII, with the exception of E188 in DI, an observation supported by our FRET data (Fig. 5h).

We tested the docking predictions by generating eleven T-AlbVFP variants, three in Site 1 (E188A, H288A, E292A) with nearby E184A as a control, and six in Site 2 (Y334A, R337A, H338A, D340A, F374A, V381A) with nearby E311A as a control. Each TAlb-VFP mutant was evaluated for its impact on the interaction with hHv1-TFP in live cells using FRET (Fig. 5i). In Site 1, the mutation H288A showed the largest effect on Alb binding; mutants E188A, E292A had moderate effects, and nearby E184A did not alter Alb binding. In Site 2, H338A had the largest effect; Y334A, R337A, D340A, and F374A had moderate effects, and both V381A and nearby E311A did not alter the binding (Fig. 5i). Thus, Alb residues H288 in Site 1 and H338 in Site 2, both in Alb DII, appeared to be fundamental to binding to hHv1.

A proposed model of Alb binding to hHvl. A structural model of the Alb-hHvl complex was generated from MD simulations using the NAMD program ${ }^{49}$, based on our knowledge of the interfacial residues identified as critical for binding by patchclamp (Fig. 4c) and FRET (Fig. 5i) experiments, the crystal structure of Alb (PDB 1BM0) ${ }^{16}$, and the EPR-derived structural model of $\mathrm{hHvl}^{43}$ (Fig. 6a and Supplementary Fig. 14). An allatom model of the transmembrane region of the dimeric hHv1 channel (residues G90 to I218) was embedded in a lipid bilayer with excess hydration. An Alb molecule was placed in the extracellular solution with the Alb-DII domain facing the extracellular surface of the channel (Supplementary Fig. 11). At the beginning of the simulation, the Alb molecule was pulled slowly toward hHv1 with distance restraints between centers of mass of side-chain heavy atoms of the highest-impact residues, that is, hHv1-H193 in each of channel subunit and Alb-H288 and AlbH338, in Sites 1 and 2, respectively. These restraints with a target distance of $5 \AA$ were gradually applied over $10 \mathrm{~ns}$ using the Colvars module in NAMD. The local residue-residue interactions in Site 1 (containing hHv1-H193 of subunit A and Alb-E188, Alb-
H288, and Alb-E292), Site 2 (involving hHv1-H193 of subunit B and Alb-Y334, Alb-R337, Alb-H388, and Alb-F374), and other contact regions of the two proteins were refined in subsequent 150 ns simulations (Supplementary Table 4). Finally, $1.5 \mu$ s long timescale ANTON2 simulation ${ }^{50}$ was performed to further relax the Alb-hHv1 complex (Supplementary Fig. 15, 16 and 17).

The final Alb-hHv1 model reveals specific interactions (Fig. 6a) consistent with the experimental observations from scanning mutagenesis of the channel (Fig. 4c) and Alb (Fig. 5i). Thus, the model exhibits electrostatic interactions in Site 1 between hHv1H193 (subunit A) and Alb-E188 and Alb-E292; and $\pi-\pi$ stacking interaction between hHv1-H193 and Alb-H288 (Fig. 6b, left). In Site 2, hHv1-H193 (subunit B) interacts with Alb-Y334, AlbR337, Alb-H338 via electrostatic and hydrogen bonding interactions and with Alb-F374 via the $\mathrm{CH}-\pi$ or $\pi-\pi$ stacking interaction (Fig. 6b, right). Here, Alb-D340 is $10 \AA$ away from hHv1-H193 (subunit $\mathrm{B}$ ), while the mechanism underlying the disruptive effect of its mutation (Fig. 5i) requires more investigation, one explanation could be that its electrostatic interaction with AlbH338 is important to the interaction of Alb-H338 and hHv1H193.

hHv1-L200 was found to influence Alb activation (Fig. 4c), but is predicted to be buried in the membrane at resting state ${ }^{4,43}$ and did not appear to be directly involved at the interaction interface in our resting state model; we suspect that mutation might change the structure of Alb-hHv1 complex in a disruptive manner as a consequence of its location in the activated channel state.

Two experiments inspired by the MD simulation results were performed and further supported the model of the Alb-hHv1 complex. First, an unanticipated hydrogen bond between AlbE382 near Site 2 and hHv1-Y134 (subunit B) was observed in the model. When the Glu residue was neutralized by mutation to alanine (producing T-Alb-E382A-VFP), the binding affinity of Alb to hHv1-TFP assessed by FRET was reduced (Fig. 5i). Second, the model identified hHv1-E192 in subunit A to be within $4 \AA$ of negatively-charged Alb-E285. Neutralizing this acidic residue in Alb (Alb-E285A-VFP) improved binding with WT hHv1 (Fig. 6i). The model further rationalized mutagenesis data that had shown neutralizing hHv1-E192 increased activation by WT Alb (Fig. 4c) by removing the destabilizing electrostatic repulsion with AlbE285.

Consistent with the notion that Alb binds to the hHv1 VSDs to activate the channels, the binding affinity of Alb was observed to increase with membrane depolarization as judged by FRET. Thus, the proton channel VSDs move "outward" to the active 
a

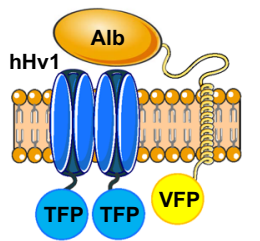

b

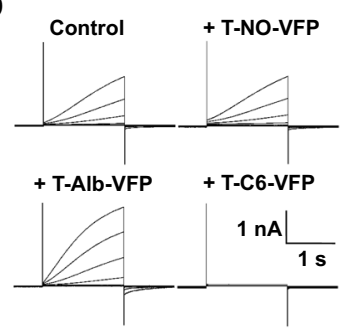

C

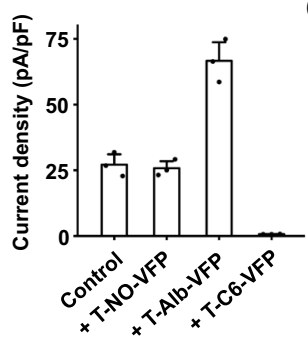

d

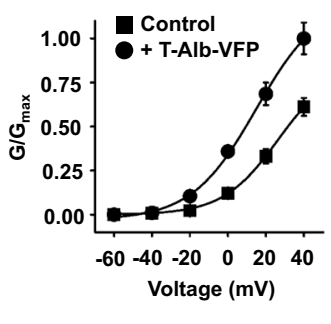

e

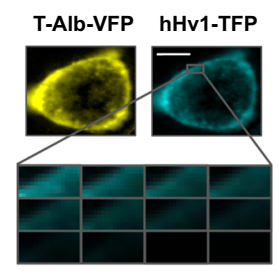

f

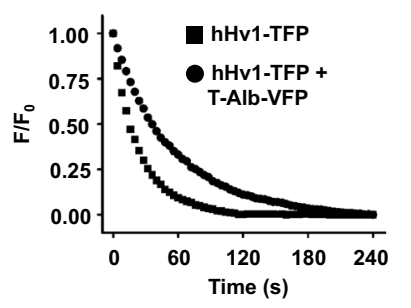

g

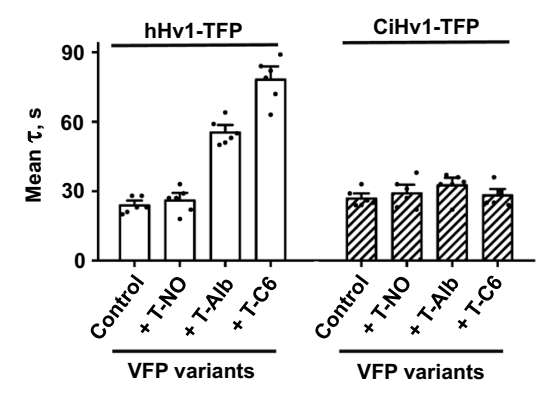

h

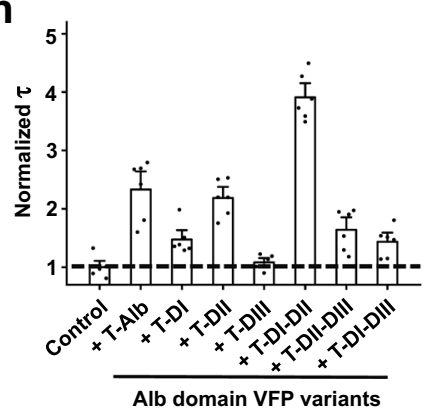

i

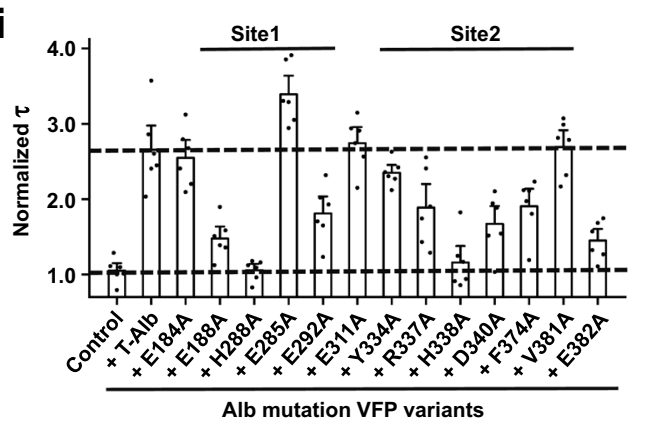

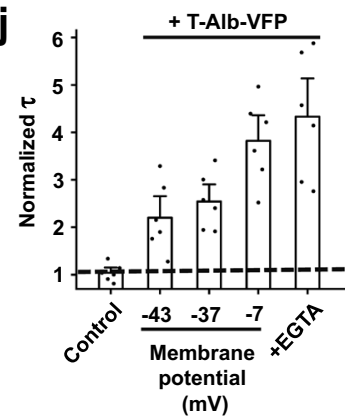

Fig. $\mathbf{5}$ DII of Alb is required for binding to hHv1 channels. Tethered constructs carrying VFP were expressed in HEK293T cells with channels tagged with TFP and studied by FRET microscopy. a Schematic representation of T-Alb-VFP showing binding of Alb to the hHv1-TFP channel. Extracellular interaction of Alb and hHv1 brought intracellular TFP and VFP into close proximity $(<10 \mathrm{~nm})$, resulting in FRET that was quantified by measuring the mean time constant $(\tau)$ for TFP (donor) photobleaching. b Representative current traces $(20 \mathrm{mV}$ steps from -60 to +40$)$ for hHv1-TFP channels expressed alone (Control) or with T-Alb-VFP, T-NO-VFP (no Alb inserted), or T-C6-VFP. c Whole-cell peak current densities of hHv1-TFP channels at $0 \mathrm{mV}$ expressed alone (Control) or with T-NO-VFP, T-Alb-VFP, or T-C6-VFP studied as in (b), $n=3$ cells. d G-V relationships for hHv1-TFP expressed alone (black squares) or with T-AlbVFP (black circles). Curves are fit to a Boltzmann equation, $n=3$ cells. e hHv1-TFP (cyan) and T-Alb-VFP (yellow) are shown to reach the cell surface. Scale bar is $10 \mu \mathrm{m}$. The boxed area of membrane shows donor photobleaching with continuous illumination. $\mathbf{f}$ Exemplar photobleaching showing the decay of fluorescence for regions of single cells expressing hHv1-TFP (black squares) or hHv1-TFP with T-Alb-VFP (black circles). $\tau$ was determined by singleexponential fits to the time course of photobleaching. $\mathbf{g}$ FRET showing hHv1-TFP (white bars) assembly with T-Alb-VFP or T-C6-VFP but not T-NO-VFP. CiHv1-TFP (white bars with black slash) ${ }^{5}$, did not show FRET with T-Alb-VFP or T-C6-VFP, $n=6$ cells. $\mathbf{h}$, Tethered Alb domain variants expressed with hHv1-TFP for FRET studies. $\tau$ of photobleaching for each pair was normalized to the $\tau$ of photobleaching of hHv1-TFP alone (Control). $n=6$ cells. $\mathbf{i}$ T-AlbVFP point mutations designed based on the two predicted interaction sites (Supplementary Fig. 13), expressed with hHv1-TFP for FRET studies. Control is hHv1-TFP expressed alone, $n=6$ cells. $\mathbf{j}$ Adjusting $\mathrm{KCl}$ in the bath by isotonic substitution for $\mathrm{NaCl}$ from $2 \mathrm{mM}$ to $10 \mathrm{mM}$ and $100 \mathrm{mM}$ changed the HEK293T cell RMP from $-43 \pm 6 \mathrm{mV}$, to $-37 \pm 4 \mathrm{mV}$ and $-7 \pm 2 \mathrm{mV}$, respectively. Increased binding of T-Alb-VFP and hHv1-TFP with membrane depolarization as demonstrated by the increasing $\tau$, from $52 \pm 7 \mathrm{~s}$ to $60 \pm 7 \mathrm{~s}$ and $90 \pm 11 \mathrm{~s}$. When $1 \mathrm{mM}$ EGTA was added to the solution with $10 \mathrm{mM} \mathrm{KCl} \tau$ was further increased to $101 \pm 15 \mathrm{~s}$. Control is hHv1-TFP expressed alone with $10 \mathrm{mM} \mathrm{KCl}, n=6$ cells. Values are mean \pm SEM. Source data are provided in the Source Data file.

conformation in response to increased membrane potential ${ }^{4,5}$ and we observe that the affinity of the interaction of T-Alb-VFP and hHv1-TFP increased as the resting membrane potential (RMP) was increasingly depolarized from $-43 \pm 6 \mathrm{mV}$ to $-7 \pm 2 \mathrm{mV}$ by isotonic replacement of $\mathrm{NaCl}$ with $\mathrm{KCl}$ in the bath solution (Fig. 5j).

\section{Discussion}

Essential role of Alb in human sperm capacitation. Two outstanding questions in sperm physiology are answered by our identification of Alb activation hHvl channels as central to initiation of capacitation. First, the RMP of non-capacitated sperm is estimated to be $-20 \mathrm{mV}$ to $-40 \mathrm{mV}^{51}$, whereas the threshold for opening of hHv1 channels was reported to be $\sim 0$ $\mathrm{mV}$ in the ionic environment present in the uterus ${ }^{4,52,53}$. This presented an enigma, how did hHv1 open under natural conditions? Here, we answer to the conundrum; because the uterus contains $\sim 500 \mu \mathrm{M}$ Alb, the $\mathrm{V}_{\text {threshold }}$ for hHv1 shifts to $\sim-30 \mathrm{mV}$ (Supplementary Fig. 10). Whereas Alb concentration is too low in semen to activate hHv1 prematurely, the level is high enough in the female reproductive tract to activate the channel and increase $\mathrm{H}^{+}$efflux, leading to intracellular alkalization and capacitation (Fig. 1, Supplementary Figs. 1 and 10) $)^{5,7}$. Second, our findings 
a

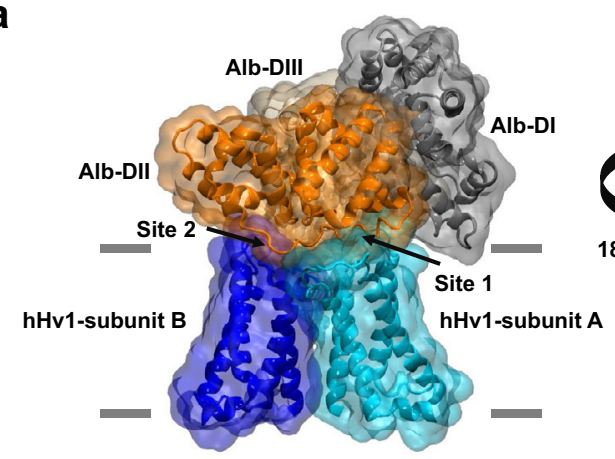

b

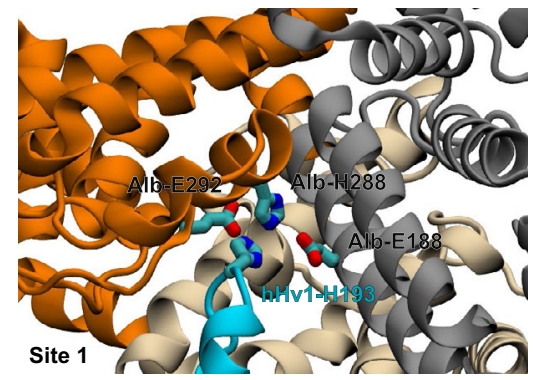

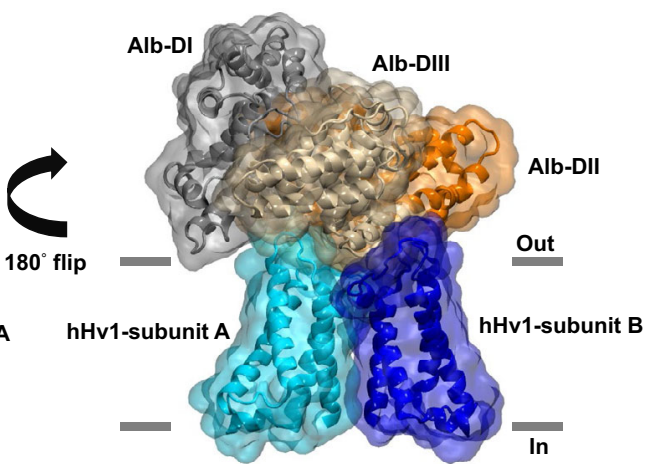

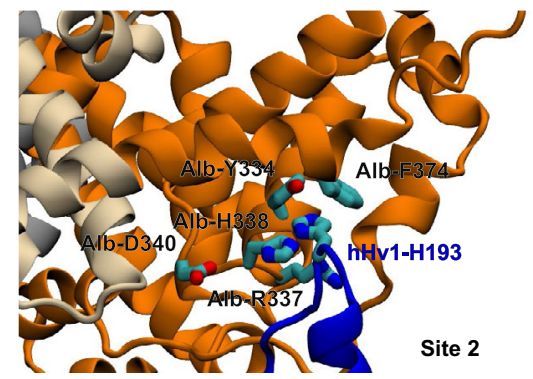

Fig. 6 A proposed Alb-hHv1 structural model from molecular dynamics simulations. a The Alb-hHv1 complex in two orientations showing the three Alb domains DI (gray), DII (orange) and DIII (wheat) and the two subunits (cyan and blue) of a dimeric hHv1 channel. The horizontal gray lines indicate the position of the membrane boundary. $\mathbf{b}$ Close-up view of the interaction interface between Alb and two individual hHv1 subunits in two binding sites in the structural model. Left, interaction Site 1, $\mathrm{H} 193$ of hHv1 subunit A (cyan) may form direct interaction with Alb residue $\mathrm{H} 288$ and the adjacent E188 and E192. Right, interaction Site 2, H193 of hHv1 subunit B (blue) may form direct interaction with Alb residue H338 and the adjacent Y334, R337, and F374.

offer a mechanistic rationale for the increase in pregnancy rates with IVF on supplementation with $\mathrm{Alb}^{19,20}$, one that does not comport with two prior hypotheses for the mechanism by which Alb might enhance oocyte fertilization.

Alb has been proposed to extract cholesterol from the sperm plasma membrane thereby allowing $\mathrm{HCO}_{3}{ }^{-}$influx to alkalinize the cells ${ }^{54}$, apparently obviating a role for $\mathrm{H}^{+}$flux via hHv1 that we demonstrated using $\mathrm{C} 6$ to block the channel ${ }^{5}$. Here, we observe that when Alb is pre-saturated with cholesterol it induces the same increase in $\mathrm{pH}_{\mathrm{i}}$ in non-capacitated human sperm as Alb alone (Supplementary Fig. 1g). Furthermore, Alb pre-saturated with cholesterol activates hHv1 channels in HEK293T cells like Alb alone (Supplementary Fig. 18a), indicating that even when Alb is unable to sequester additional cholesterol it can activate the channel to achieve sperm alkalization. We support an alternative explanation for the loss of cholesterol from sperm during capacitation. Since the equilibrium level of $\mathrm{HCO}_{3}{ }^{-}$in cells is a function of total carbon concentration and $\mathrm{pH}^{55}$, alkalization caused by increased $\mathrm{H}^{+}$efflux via hHvl will increase intracellular $\mathrm{HCO}_{3}{ }^{-}$and this has been demonstrated by others to activate adenylyl cyclase and protein kinase A, upregulating phospholipid scramblases that facilitate cholesterol loss from sperm ${ }^{27,56}$.

A second hypothesis for augmented fertilization success with Alb has been that it sequesters $\mathrm{Zn}^{2+}$, decreasing the already low concentration of $\mathrm{Zn}^{2+}$ in the uterus compared to semen ${ }^{57}$, thereby releasing $\mathrm{hHv} 1$ from residual $\mathrm{Zn}^{2+}$ inhibition ${ }^{7}$. While such a role for Alb remains possible, we observe that Alb has similar stimulatory effects on hHv1-H140A and WT hHv1 channels (Supplementary Fig. 18b, c), although the mutant has an $\sim 30$-fold lower affinity for $\mathrm{Zn}^{2+}$ than WT channels ${ }^{2}$. This shows that hHv1 activation by Alb does not require $\mathrm{Zn}^{2+}$ chelation.

Furthermore, hHv1 activation by Alb does not appear to result from chelation of trace elements. It has become commonplace to add EDTA or EGTA to bath solutions for biophysical studies of
hHv1 because this speeds current activation, slows deactivation, and shifts the $\mathrm{G}-\mathrm{V}$ relationships to more hyperpolarized potentials, increasing the current magnitude so the channel is easier to study ${ }^{4}$. The effects of EDTA and EGTA have been attributed to chelation of trace heavy metals contaminating salt solutions ${ }^{4}$. We do not support the notion that Alb activates hHv1 by chelation of trace metals, first, because Alb activation is increased (or decreased) by mutating sites on the channel (Fig. 4c) or Alb (Fig. 5i) that mediate Alb-hHv1 binding. Further, we presented two examples of mutations at the Alb-hHv1 binding interface that are suppressed by secondary mutations on the other protein (hHv1-Y134 and Alb-E382; hHv1-E192, and Alb-E285, Fig. 6i) and it seems unlikely that each mutation, and restoring counter mutation, would modify heavy metal binding by Alb especially those on the channel. Finally, the hyperpolarizing shift in the $\mathrm{V}_{1 / 2}$ of hHv1 in sperm induced by EDTA is far less than that produced by Alb. Thus, the $\mathrm{V}_{1 / 2}$ in the absence of both EDTA and Alb is $\sim 15 \mathrm{mV}$, addition of $500 \mu \mathrm{M}$ Alb produces a negative shift of $53 \mathrm{mV}$ to $-38 \mathrm{mV}$, whereas $1 \mathrm{mM}$ EDTA alone produces a shift only to $4 \mathrm{mV}$ (Supplementary Table 5, and Supplementary Fig. 19); further suggesting that they act differently, adding both Alb and EDTA produces a smaller shift, to $\mathrm{V}_{1 / 2}=-28 \mathrm{mV}$, than Alb alone.

Similar to what we observed in sperm, $500 \mu \mathrm{M}$ Alb alone shifts the $\mathrm{V}_{1 / 2}$ of cloned hHv1 channels in HEK293T cells $-45 \mathrm{mV}$ (Supplementary Table 5). However, in contrast to sperm, EDTA and EGTA equally effective as Alb on the heterologouslyexpressed channel, inducing a shift approximately $-40 \mathrm{mV}$. Of note, the interaction of T-Alb-VFP and hHv1-TFP measured by FRET increases on application of $1 \mathrm{mM}$ EGTA, in the same fashion as membrane depolarization (Fig. 5j). This suggests that EGTA, like Alb, favors the outward configuration of the hHv1 VSDs to increase Alb binding, shifting the $\mathrm{V}_{1 / 2}$ so far that Alb has no further effect (Supplementary Fig. 20). The partial impact of 
EGTA/EDTA on sperm $\mathrm{H}^{+}$current compared to Alb (versus the homologous impact on the cloned channel in HEK293T cells) suggests we do not fully understand how the chemical chelators alter hHv1 function and invites further exploration.

Alb potentiation of $\mathrm{hHvl}$ is established here as a required and natural mechanism for capacitation initiation by three observations (Fig. 7a). First, our prior demonstration that block of hHv1 by $\mathrm{C} 6$ inhibits capacitation (Fig. 1 and Supplementary Fig. 1) $)^{5}$. Second, our demonstration here that direct binding of Alb to hHv1 shifts the $\mathrm{V}_{\text {threshold }}$ so the channel is active at the hyperpolarized membrane potentials measured in human sperm (Figs. 1, 3 and Supplementary Fig. 3, 10). And, third, that Alb concentration in the uterus, but not semen, is sufficient to activate hHv1 (Fig. 1).

Alb modulates the innate immune system via hHvl. Neutrophils are the most abundant white blood cell type and an essential part of the innate immune system that defends against invading pathogens via ROS release, a process that is also implicated in pathogenesis related to oxidative stress ${ }^{32}$. When stimulated, neutrophil NOX2 produces ROS by reducing $\mathrm{O}_{2}$ to $\mathrm{O}_{2}{ }^{--}$, a reaction that translocates electrons across the plasma membrane, depolarizing cells, and generating protons in the cytoplasm that inhibit further NOX2 activity 23,33 . Proton efflux via Hv1 allows sustained ROS production by NOX2 by maintaining appropriate membrane potential and $\mathrm{pH}_{\mathrm{i}}^{8,10}$, as demonstrated by genetic deletion of Hv1 in mice ${ }^{9}$ and by C6 blockade in human neutrophils 5 .

In this study, we used fMLF to induce the respiratory burst in human neutrophils and show that Alb acts via hHvl to enhance NOX2 activity. Indeed, Alb was previously observed to increase ROS production by neutrophils although the relevant receptor and mechanism were unknown ${ }^{22}$. The importance of Alb potentiation of hHv1 in neutrophils can be inferred from estimates that during the respiratory burst the cells would depolarize from roughly $-60 \mathrm{mV}^{58}$ to $+200 \mathrm{mV}$ within $20 \mathrm{~ms}$ without charge compensation by $\mathrm{hHvl}^{34}$, and that activation of $\mathrm{hHvl}$ is slower in the absence of Alb (Figs. 2a and 3a). Moreover, it is projected that NOX2 activity without offset would decrease $\mathrm{pH}_{\mathrm{i}}$ by more than five units based on the amount of $\mathrm{O}_{2} \cdot{ }^{-}$ generation ${ }^{59}$. Potentiated by Alb, hHv1 opens at more negative membrane potentials and faster allowing for more effective compensation. Of note, as the concentration of stimulatory fMLF is increased, the potentiating effect of Alb increases to meet the higher demand for compensation (Fig. 2c, Supplementary Fig. 3d).

A number of the attributes of the neutrophil response appear to protect against aberrant inflammatory mediator release. Alb in the absence of a pathogenic stimulus does not induce ROS production (Supplementary Fig. 3b). Furthermore, the level of Alb varies in different tissues ${ }^{14}$ but it averages $\sim 30 \mathrm{mg} / \mathrm{mL}$ ( 450 $\mu \mathrm{M})$ in many compartments. This Alb concentration will shift the a

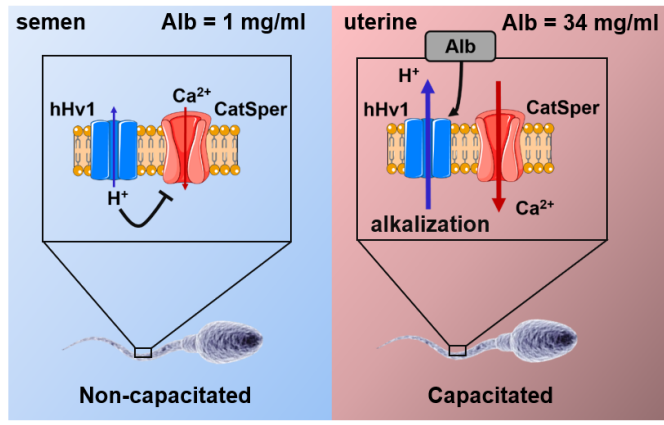

b

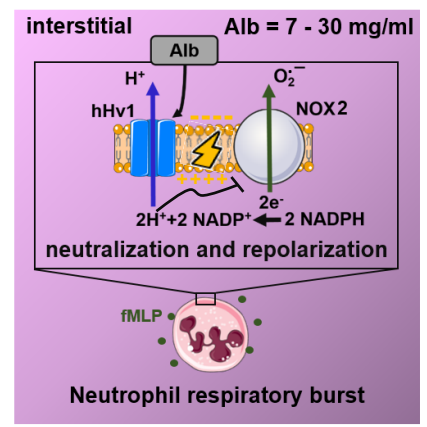

C

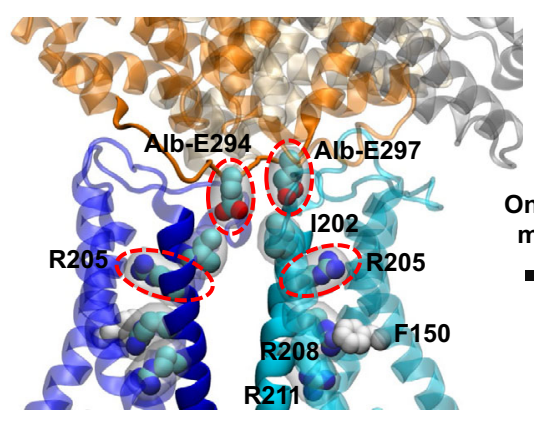

Alb-hHv1 at channel resting state

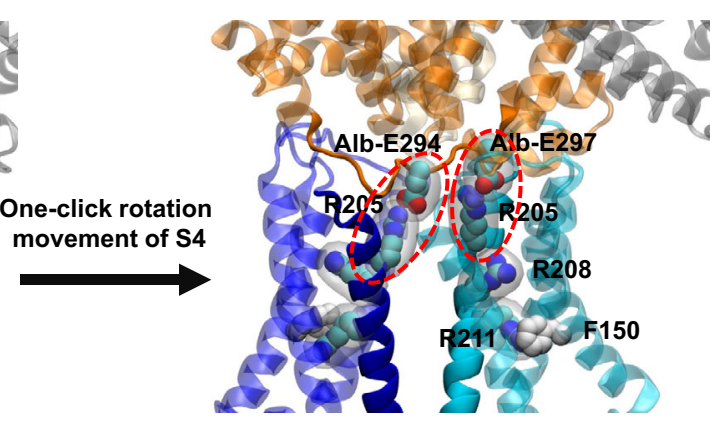

Alb-hHv1 at channel activated state

Fig. 7 Physiology of Alb activation of hHv1 in human sperm and neutrophils and proposed molecular mechanism. a The higher level of Alb in the female reproductive tract activates $\mathrm{hHv} 1$ in sperm leading to $\mathrm{H}^{+}$efflux and cytosolic alkalization, initiating capacitation by potentiating CatSper. The synergy of hHv1 and CatSper in human sperm capacitation has previously been described ${ }^{5-7}$. b Activation of $\mathrm{hHv1}$ in neutrophils by $\mathrm{Alb}$ potentiates $\mathrm{H}^{+}$efflux, maintaining physiological $\mathrm{pH}_{\mathrm{i}}$ and compensating for the depolarization caused by NOX2, enhancing enzyme activity and allowing sustained ROS production. During the neutrophil respiratory burst (here, stimulated by fMLF), NOX2 translocates electrons from NADPH across the membrane to reduce $\mathrm{O}_{2}$ to $\mathrm{O}_{2}{ }^{-\cdots}$; this efflux of electrons generates $\mathrm{H}^{+}$in the cytoplasm and depolarizes cells, inhibiting the activity of NOX2 $2^{5,8-10}$. c Alb activation of hHv1 is proposed to proceed by an electrostatic gating modification mechanism. Two binding sites in the Alb DII domain interact with the two S3-S4 loops in hHv1 channels. The two Alb sites each have an adjacent negatively-charged residue (Alb-E294 and Alb-E297) that attracts the first channel gating charge (hHv1R205) to facilitate outward movement of the voltage sensors and stabilizes the sensors in the activated "up" conformation. Side chains of three gating charges (R205, R208, and R211), the "hydrophobic gasket" formed by F150 that separates the internal and external aqueous solutions ${ }^{73}$, and I202, one helical turn above R205, are shown on hHv1 subunit A (cyan). 
$\mathrm{V}_{\text {threshold }}$ of $\mathrm{hHvl}$ approximately $-30 \mathrm{mV}$ (Supplementary Fig. 3b), therefore is estimated to bring the neutrophil hHv1 $\mathrm{V}_{\text {threshold }}$ in native environment $\left(\mathrm{pH}_{\mathrm{i}}=7.3, \mathrm{pH}_{\mathrm{o}}=7.4\right)$ from $\sim 20$ $\mathrm{mV}$ to $-10 \mathrm{mV}$, a level still far above the RMP of approximately $-60 \mathrm{mV}^{58}$ until immune stimulation produces a respiratory burst that depolarizes the cells. Indeed, in many tissue compartments Alb is low in health but increases with disease, for example, the hallmark of infectious acute respiratory distress syndrome in the lung is accumulation Alb-rich edema and neutrophilic infiltrates in the air-spaces ${ }^{60}$. The presence of Hv1 in mouse neutrophils has been shown to facilitate $\mathrm{Ca}^{2+}$ influx ${ }^{10}$, and this is required for elastase release ${ }^{61}$. This may explain why the action of Alb on hHv1 also increases elastase release from human neutrophils (Fig. 2d). Our findings demonstrate that Alb is an innate immune system regulator and identifies potentiation of hHv1 as a previously unrecognized innate immunity mechanism that enhances the inflammatory activity of neutrophils in health and disease (Fig. 7b).

A physical model for Alb binding on hHvl. Given the critical physiological roles demonstrated for Alb via hHv1, and in the absence of high resolution atomic structural information, we judged it valuable and feasible to construct a structural model of the Alb-hHv1 macromolecular complex. The Alb-hHv1 model was constructed by MD simulations (Fig. 6a), and evaluated experimentally using $\mathrm{Alb}$ and Alb variants tethered to the extracellular surface of the cells expressing hHv1 or hHvl variants (Fig. 5h, i), a strategy previously applied to peptide neurotoxins to identify residues important in toxin-channel complexes ${ }^{46}$. The model locates hHv1-H193 of subunit A in Site 1 with Alb-H288, and hHv1-H193 of subunit B in Site 2 with Alb-H338 (Fig. 6b). These interactions appear to coordinate Alb binding to the two voltage-sensor loops in hHv1 channels (Fig. 6a, b), while other Alb residues (E188, E292, Y334, R337 and F374) contribute to the two sites through a complex network of residue-residue interactions (Fig. 6b). The model rationalizes why neutralizing hHv1E192 in the channel improves WT Alb activation (Fig. 4c). We consider limitations of our modeling approach, including the persistence of restraints during long-term simulations and our inability to simulate the complete binding/unbinding process due the large size of the system ( 185,000 atoms) in Supplementary Fig. 14.

A proposed mechanism, Alb as an electrostatic gating modifier. Some neurotoxins target VGICs to activate or inhibit the channels by favoring the "up" or "down" state of the voltage sensors, respectively. By this mechanism CssIV activates Nav1.2, while C6 inhibits hHv1 ${ }^{5,62}$. We suggest that the physiological effects of Alb on hHv1 take place by a similar mechanism, whereby Alb favors the activated position of the two hHvl voltage sensors.

hHv1 responds to membrane potential changes via three arginine residues, R205, R208 and R211 in the S4 helix (Fig. 7c) ${ }^{4}$. The residues move in the electric field facilitated by interactions with acidic residues in the neighboring S1-S3 helices ${ }^{13}$. Recently, a $\mu$ s timescale $\mathrm{MD}$ simulation of hHv1 suggested that depolarization moves the S4 helix upward, placing R205 at the polar-apolar membrane surface near D123 in the S1 helix ${ }^{63}$. In our model of the hHv1 resting state, the $\mathrm{S} 4$ helices are in the "down" position, placing hHv1-I202 (one helical turn above the first gating charge hHv1-R205) in channel subunits A and B toward Alb-E294 and Alb-E297 of Alb DII, respectively. We posit that upon depolarization, the electrostatic attraction between the two hHv1-R205 residues and the two negative Alb residues, AlbE294 and Alb-E297, lowers the energy for the upward movement of the S4 helices, accelerating and sustaining the activation of hHv1 (Fig. 7c).

Supporting the notion that Alb acts as a gating modifier, it shifts the activation of hHvl to more negative potentials (Fig. $3 \mathrm{~b}$ and Supplementary Fig. 5b) and speeds channel activation (Fig. 3a and Supplementary Fig. 5a). Also supporting this mechanism, the affinity of Alb for hHvl was observed to increase with depolarization (Fig. 5j and Supplementary Fig. 5c). Finally, we highlight the fact that neutralizing the channel residue to produce hHv1-R205N yields a channel that is insensitive to Alb (Supplementary Fig. 21a), whereas hHv1-R211S channels were activated like WT (Supplementary Fig. 21b). This is in agreement with the molecular mechanism we propose based on a closed state model where hHv1-H193 in each subunit interacts with Alb$\mathrm{H} 288$ or Alb-H338, and an open state model where the "up" sensor position allows the first gating charge in each channel subunit (hHv1-R205) to interact with Alb-E294 and Alb-E297, favoring the active channel conformation and potentiated proton fluxes.

hHv1 is implicated in a wide range of biological processes in addition to the capacitation of sperm and the innate immune responses we study here ${ }^{4}$. The channels have some notable roles in proliferation of cancer cells ${ }^{24}$, tissue damage during ischemic stroke $^{64}$, and hypertensive injury of the kidney ${ }^{65}$. Because Alb is ubiquitous at levels that vary in different human compartments in health and disease ${ }^{14}$, the potentiation of hHvl by Alb we describe in this report will be wide-spread, tissue-dependent, and play both salutary and unfavorable roles in human physiology.

\section{Methods}

Molecular biology. The coding sequences for human Hv1 (NM_001040107) or Ciona intestinalis Hv1 (NM_001078469) were tagged with a teal fluorescent protein (TFP) via a 13-residue linker using gBlock gene fragments (Integrated DNA Technologies) and inserted into a laboratory dual-purpose vector pMAX using Gibson Assembly (New England BioLabs). $\mathrm{h}_{\mathrm{S} 1-\mathrm{S} 2}$, s3-S4 CiHv1 was constructed by replacing the nucleotides for the S1-S2 loop (L169-L187) and S3-S4 loop (G231L254) of CiHv1 with S1-S2 loop (I121-F139) and S3-S4 loop (I183-L204) of hHv1. Similarly, $\mathrm{h}_{\mathrm{S} 1-\mathrm{S} 2} \mathrm{CiHv} 1$ was constructed by replacing the S1-S2 loop (L169-L187) of CiHv1 with S1-S2 loop (I121-F139) of hHv1; $\mathrm{h}_{\mathrm{S} 3-\mathrm{S} 4} \mathrm{CiHv} 1$ was constructed by replacing the S3-S4 loop (G231-L254) of CiHv1 with S3-S4 loop (I183-L204) of $\mathrm{hHv1}$; and, $\mathrm{Ci}_{\mathrm{S} 3-\mathrm{S} 4} \mathrm{Hv} 1$ was constructed by replacing the S3-S4 loop (I183-L204) of hHv1 with S3-S4 loop (G231-L254) of CiHv1. All chimeras were assembled into pMAX using Gibson assembly. Hv1Sper was constructed by truncating 67 residues at the N-terminus of hHv1 (residues from A2 to R68). hKv1.3 (NM_002232), hKv1.5 (NC_000012), hKv2.1 (NP_004966), hKCNQ1 (NM_000218), hKCNE1 (NP_000210.2), hNav1.5 (NC_000003) and the sodium voltage-gated channel $\beta$ subunit 1 (NC_000019) in pMAX were used as previously described. Tethered Albumin (Alb) constructs were built in pMAX vector. To generate T-Alb-VFP, the PDGF-receptor transmembrane domain was amplified and inserted into pMAX vector. Subsequently, sequences encoding the preprotrypsin secretory pathway signal sequence (secretion signal), Alb sequence and a 16 residues flexible linker region (Gly-Asn) x 8 were inserted at the $5^{\prime}$ end of the PDGF transmembrane domain. Fluorescent protein mVenus (VFP) was attached to the C-terminus of transmembrane domain. T-C6-VFP with C6 sequence replacing Alb and T-NOVFP without Alb sequence incorporation was constructed using the same strategy. Tethered Alb domain constructs with sequence insertion of DI (residues 1-194), DII (residues 183-387), DIII (residues 381-585), DI-DII (residues 1-387), DII-DIII (residues 183-585), and DI-DIII (residues 1-194 and 381-585, respectively) were generated $^{47}$ using PCR and then ligated into pMAX vector. Point mutations for hHvl and T-Alb-VFP were introduced using QuikChange Site-Directed Mutagenesis Kit (Agilent). Primers used to generate point mutations and tethered Alb domain constructs are summarized in Supplementary Table 6. The sequences of all constructs were confirmed by DNA sequencing. Because hHv1 with V187C, L189C and L203C changes did not have expression in HEK293T cells, alanine mutations were studied at these three sites.

Cell culture. HEK293T cells (RRID: CVCL_0063) were obtained from ATCC and used for heterologous expression. Cells were maintained in Dulbecco's Modified Eagle Medium (DMEM) (ATCC) supplemented with $10 \%$ fetal bovine serum and $1 \%$ penicillin and streptomycin (Gibco) and incubated at $37^{\circ} \mathrm{C}$ in a humidified atmosphere containing $5 \% \mathrm{CO}_{2} / 95 \%$ air. Plasmids were transfected into cells using Lipofectamine 2000 (Life Technologies) according to the manufacturer's instructions. Experiments were preformed $24-48 \mathrm{~h}$ post transfection. 
Proteins, peptides and reagents. C6 toxin (MH828728) was purchased as synthetic peptides from CSBio. Peptide toxin folding reactions were quenched by acidification and purified by reverse-phase HPLC, as before ${ }^{5}$. Peptides that were more than $95 \%$ pure were lyophilized and stored at $-20^{\circ} \mathrm{C}$. The composition of the peptides was confirmed by mass spectral analysis. Peptides were dissolved in appropriate external solutions for whole-cell patch clamp recordings or physiological assays before use. Albumin from human serum (lyophilized powder, fatty acid free) was purchased from Sigma (A1887). Proteinase K (Lyophilized) was purchased from Promega (V3021) and the digestion was performed following the protocol supplied with the product. Purified Fab fragment of human IgG was purchased from GenWay Biotech (GWB-DD0665). Alb was labeled with 5,6TAMRA-SE (5-(and-6-)-carboxytetramethyl-rhodamine succinimidyl ester) (Life Technologies) per manufacturer instructions. Thus, Alb was dissolved in $50 \mathrm{mM}$ HEPES, $100 \mathrm{mM} \mathrm{NaCl}$ at $\mathrm{pH} 7.5$ to a concentration of $10 \mathrm{mg} / \mathrm{mL}$. 5,6-TAMRA-SE dye was dissolved in $500 \mu \mathrm{L}$ DMSO. Alb and dye were mixed at a molar ratio of 1:10 for $1 \mathrm{~h}$ at room temperature on a rotating shaker. The labeled Alb was purified by HPLC over a $20-80 \%$ acetonitrile gradient and the corresponding single peak was collected. Samples were lyophilized and stored at $-80^{\circ} \mathrm{C}$. Labeled protein was dissolved in appropriate external solution before use. All the reagents and chemicals were purchased from Sigma-Aldrich unless otherwise stated.

Human sperm preparation. Ejaculates were obtained from healthy donors by masturbation after at least $48 \mathrm{~h}$ of sexual abstinence. Only semen samples that fulfilled the World Health Organization (WHO 2010) guidelines were selected for experiments. All semen donors gave written informed consent and the protocol for semen sample handling were approved by the Ethic Committee of the School of Medicine, National University of Cuyo and the Bioethics Committee at the Biotechnology Institute from the National Autonomous University of Mexico. After sample liquefaction $\left(30 \mathrm{~min}\right.$ at $\left.37^{\circ} \mathrm{C}\right)$, motile sperm were recovered after a swimup separation for $1 \mathrm{~h}$ at $37^{\circ} \mathrm{C}^{5}$.

Human peripheral blood neutrophils purification. Human polymorphonuclear neutrophils were isolated from peripheral blood from healthy donors by FicollPaque Plus (GE Healthcare) density-gradient centrifugation. Peripheral blood was obtained from Institute for Clinical and Translational Science of University of California Irvine and the protocol was approved by the Institutional Review Board of University of California Irvine. Donor population is composed of 50\% female and $50 \%$ male with ages ranging from 23 to 62 years old. $20 \mathrm{~mL}$ blood was mixed with 3\% dextran in PBS (Sigma-Aldrich) for $20 \mathrm{~min}$ in a $50 \mathrm{~mL}$ conical tube. The top clear layer containing leukocytes was collected and underlaid with $10 \mathrm{~mL}$ of Ficoll-Paque Plus. The cell suspension was centrifuged at $500 \times g$ for $30 \mathrm{~min}$ at $20^{\circ} \mathrm{C}$ to separate mononuclear cells from neutrophils and the remaining red blood cells. The overlying plasma and monocyte layers were aspirated, and the neutrophils and red blood cells pellet was re-suspended in Red Blood Cells Lysis Buffer (eBioscience), incubated for $10 \mathrm{~min}$ to lyse red blood cells. In total, $35 \mathrm{~mL}$ PBS was added to stop the lysis and the cell suspension was centrifuged at $300 \times g$ for $5 \mathrm{~min}$ at $4{ }^{\circ} \mathrm{C}$. Cell pellet was re-suspended in RPMI1640 (Gibco). An aliquot of neutrophils was mixed with Trypan blue (Gibco) and counted using a hemocytometer. Neutrophils isolated using this method were routinely found to be greater than $97 \%$ of the final cell preparation.

Electrophysiology. Proton current from hHvl, CiHvl and chimera channels were recorded in whole-cell mode using an Axopatch 200B amplifier. Stimulation and data collection were achieved with a Digidata1440A and pCLAMP 10 software (Molecular Devices). HEK293T cells expressing hHv1 channel variants were perfused with a nominally divalent cation-free external solution of $100 \mathrm{mM}$ HEPES, $100 \mathrm{mM} \mathrm{NaCl}, 10 \mathrm{mM}$ glucose at $\mathrm{pH} 7.5$ or human tubular fluid medium (HTF) comprising $101.6 \mathrm{mM} \mathrm{NaCl}, 4.69 \mathrm{mM} \mathrm{KCl}, 0.2 \mathrm{mM} \mathrm{MgSO}_{4}, 0.37 \mathrm{mM} \mathrm{KH}_{2} \mathrm{PO}_{4}$, $2.04 \mathrm{mM} \mathrm{CaCl}_{2}, 25 \mathrm{mM} \mathrm{NaHCO}, 2.78 \mathrm{mM}$ Glucose, $0.33 \mathrm{mM}$ Na pyruvate and $21.4 \mathrm{mM} \mathrm{Na}$ lactate at $\mathrm{pH} 7.2$, unless otherwise noted. Pipettes with resistances between 3-5 M $\Omega$ were filled with $100 \mathrm{mM}$ Bis-Tris buffer, $100 \mathrm{mM} \mathrm{NaCl}$, and 10 $\mathrm{mM}$ glucose at $\mathrm{pH} 6.5$ or 6.7. For EGTA recordings, 1 mM EGTA was added in the external solution with the supplement of $2 \mathrm{mM} \mathrm{MgCl}_{2}$. Sampling frequency was 10 $\mathrm{kHz}$ and was filtered at $1 \mathrm{kHz}$. Alb was applied in the external solution through a multichannel micro-perfusion system after currents monitored by test pulses to 0 $\mathrm{mV}$ for $1.5 \mathrm{~s}$ from a holding voltage of $-60 \mathrm{mV}$, with $10 \mathrm{~s}$ interpulse intervals, were judged to be stable. Current-voltage relationships were evoked from a holding potential of $-60 \mathrm{mV}$ to test pulses from $-60 \mathrm{mV}$ to $+60 \mathrm{mV}$ for $1.5 \mathrm{~s}$ in $20 \mathrm{mV}$ intervals every $10 \mathrm{~s}$.

Proton currents in human neutrophils were recorded with an external solution of $100 \mathrm{mM}$ HEPES, $130 \mathrm{mM}$ NMDG, $10 \mathrm{mM}$ glucose at $\mathrm{pH}$ 7.5. Pipettes with resistances between 10 and $15 \mathrm{M} \Omega$ were filled with $100 \mathrm{mM}$ MES buffer, $130 \mathrm{mM}$ NMDG, and $1 \mathrm{mM}$ EGTA at $\mathrm{pH}$ 6.0. Current-voltage relationships were evoked from a holding potential of $-60 \mathrm{mV}$ to test pulses from $-60 \mathrm{mV}$ to $+60 \mathrm{mV}$ for $5 \mathrm{~s}$ in $20 \mathrm{mV}$ intervals every $15 \mathrm{~s}$. Current was assessed at the end of the test pulse.

The G-V relationships were determined as described by DeCoursey ${ }^{66}$, the reversal potential $\left(\mathrm{V}_{\text {rev }}\right)$ is calculated with the equation $\mathrm{V}_{\text {rev }}=\left(\mathrm{I}_{\text {end }}-\mathrm{I}_{\text {tail }}\right) /\left(\mathrm{V}_{\text {test }}-\right.$ $\left.\mathrm{V}_{\text {hold }}\right)$, and were fit to the Boltzmann equation, $\mathrm{G}=\mathrm{G}_{\max } /\left[1+\exp \left(-\mathrm{zF}\left(\mathrm{V}-\mathrm{V}_{1 / 2}\right) /\right.\right.$ RT)], where $G_{\max }$ is maximum conductance, $V$ is the test potential, $V_{1 / 2}$ is the voltage of half-maximal activation, $\mathrm{z}$ is the effective valence, $\mathrm{T}$ is the temperature, $\mathrm{R}$ is the gas constant, and F is the Faraday constant. Deactivation kinetics for hHvl with and without Alb were determined by fitting traces with single exponential functions. Activation kinetics were fit with a single exponential having a delay. $k_{\mathrm{on}}$ and $k_{\text {off }}$ were estimated from fits of the kinetics of toxin wash-in and wash-out and calculated using equations:

$$
\tau_{\text {on }}=\left(k_{\text {on }}[\mathrm{Tx}]+k_{\text {off }}\right)^{-1}
$$

and

$$
\tau_{\text {off }}=\left(k_{\text {off }}\right)^{-1}
$$

A dose-response curve was determined by normalizing the current or the time constants of channel activation $\left(\tau_{\text {act }}\right)$ to the values before the application of Alb, then plotting versus concentration of Alb. Dose-response curves were fitted with a Hill equation (Eq. (3)) in Origin 8.0.

$$
\mathrm{r}=[\mathrm{Alb}]^{\mathrm{h}} /\left(\mathrm{EC}_{50}{ }^{\mathrm{h}}+[\mathrm{Alb}]^{\mathrm{h}}\right)
$$

where $r$ is the rate of hHv1 current increasing with Alb at equilibrium, [Alb] is the concentration of Alb, and $\mathrm{h}$ is the Hill coefficient. The equilibrium affinity $\left(K_{d}\right)$ of Alb for hHvl binding was estimated similarly by Hill equation, using the rate of hHv1 $\tau_{\text {act }}$ increasing and assuming Hill coefficient is 1 (simple bimolecular interaction).

Perforated patch clamp was performed with nystatin at $150 \mu \mathrm{g} / \mathrm{ml}$ in the pipette solution. After seal formation, the resting membrane potential of cells expressing $\mathrm{hHv1}$ channels was measured in current-clamp configuration after attainment of whole-cell configuration with $10 \mathrm{mM}$ HEPES, $136 \mathrm{mM} \mathrm{KCl}, 1 \mathrm{mM} \mathrm{MgCl} 2,2 \mathrm{mM}$ $\mathrm{K}_{2} \mathrm{ATP}, 5 \mathrm{mM}$ EGTA, pH 7.2 in the pipette and the bath solution described in the section Live cell FRET microscopy.

Xenopus laevis oocytes were injected with cRNA encoding hHvl and proton current was measured using two-electrode voltage clamp (TEVC) three days thereafter. Recording solution for hHvl was $60 \mathrm{mM} \mathrm{NaCl}, 1 \mathrm{mM} \mathrm{MgCl}, 2 \mathrm{mM}$ $\mathrm{CaCl}_{2}, 120 \mathrm{mM}$ HEPES, $\mathrm{pH}$ 7.3. Before hHv1 recording, we injected oocytes with $50 \mathrm{~nL}$ of $1 \mathrm{M}$ HEPES ( $\mathrm{pH}=7.3$ ) to minimize $\mathrm{pH}$ changes due to proton efflux. This results in $\sim 100 \mathrm{mM}$ HEPES in the cytosol. For recording the potassium currents, Xenopus laevis oocytes were injected with cRNA encoding $\mathrm{hKv} 2.1$ or $\mathrm{h}_{\mathrm{S3}}$ ${ }_{54} \mathrm{Kv} 2.1$. Recording solution was composed of $50 \mathrm{mM} \mathrm{KCl}, 50 \mathrm{mM} \mathrm{NaCl}, 1 \mathrm{mM}$ $\mathrm{MgCl} 2,0.3 \mathrm{mM} \mathrm{CaCl}$, $10 \mathrm{mM}$ HEPES, $\mathrm{pH}$ 7.5. Recordings were performed with constant gravity flow of solution at $2 \mathrm{~mL} / \mathrm{min}$ yielding chamber exchange in $\sim 5 \mathrm{~s}$. Currents were recorded 1-2 days after cRNA injection using an Oocyte clamp amplifier OC-725C (Warner Instruments, Hamden, CT), and electrodes filled with $3 \mathrm{M} \mathrm{KCl}$ with resistance of $0.3-1 \mathrm{M} \Omega$. Data were filtered at $1 \mathrm{kHz}$ and digitized at $20 \mathrm{kHz}$ using pCLAMP 10 and assessed with Clampfit 10 and Origin 8.

Procedures of human sperm electrophysiology were approved by the Bioethics Committee at the Biotechnology Institute from the National Autonomous University of Mexico. Motile sperm were recovered after a swim-up separation for $1 \mathrm{~h}$ in modified Krebs-Ringer bicarbonate medium under non-capacitation conditions (without BSA and $\mathrm{Ca}^{2+}$ ) at $37^{\circ} \mathrm{C}$ in a humidified atmosphere of $5 \%$ $\mathrm{CO}_{2}-95 \%$ air. Spermatozoa were stored in physiological solution comprising 135 $\mathrm{mM} \mathrm{NaCl}, 5 \mathrm{mM} \mathrm{KCl}, 1 \mathrm{mM} \mathrm{MgSO}_{4}, 2 \mathrm{mM} \mathrm{CaCl}_{2}, 5 \mathrm{mM}$ glucose, $1 \mathrm{mM}$ sodium pyruvate, $10 \mathrm{mM}$ lactose and $20 \mathrm{mM}$ HEPES, pH 7.4 until used in electrophysiological recordings. Whole-cell patch clamp was used to record proton currents sealing at the cytoplasmic droplet from mature human spermatozoa plated on poly-lysine coated coverslips. Pipettes $(20-30 \mathrm{M} \Omega)$ were filled with $135 \mathrm{mM} \mathrm{N}$ methyl-D-glucamine (NMDG), $5 \mathrm{mM}$ ethylene glycol tetraacetic acid (EGTA), and $100 \mathrm{mM}$ MES adjusted to $\mathrm{pH} 6.0$ with methanesulfonic acid $\left(\mathrm{CH}_{3} \mathrm{SO}_{3} \mathrm{H}\right)$. Seals between the patch pipette and the sperm cytoplasmic droplet were formed in physiological solution. After transition into whole-cell mode, the bath solution was changed to one that was free of added divalent cations comprised of $130 \mathrm{mM}$ NMDG, $1 \mathrm{mM}$ EDTA and $100 \mathrm{mM}$ HEPES, pH 7.4 with $\mathrm{CH}_{3} \mathrm{SO}_{3} \mathrm{H}$ to measure dose-dependent activation of proton currents by Alb (Fig. 1a, b) and the G-V shift after application of $800 \mu \mathrm{M}$ Alb (Fig. c). To determine the absolute effect of Alb on the G-V shift of sperm proton currents, we used the same bath solution without 1 mM EGTA (Supplementary Fig. 19 and Supplementary Table 1). CatSper currents were recorded in divalent cation-free solutions that contained $150 \mathrm{mM}$ sodium gluconate, $2 \mathrm{mM} \mathrm{Na}_{2}$ EDTA, $2 \mathrm{mM}$ EGTA, $20 \mathrm{mM}$ HEPES, pH 7.4, and the pipette solution contained $135 \mathrm{mM} \mathrm{Cs}-\mathrm{MeSO}_{3}, 5 \mathrm{mM} \mathrm{CsCl}, 5 \mathrm{mM}$ Na-ATP, $10 \mathrm{mM}$ EGTA, $20 \mathrm{mM}$ HEPES, pH 7.0. For CatSper current recordings we used a conventional voltage-ramp protocol from $-80 \mathrm{mV}$ to $+80 \mathrm{mV}$ lasting $750 \mathrm{~ms}$ from a holding potential of $0 \mathrm{mV}$. Pulse protocol application and data acquisition were performed with a patch-clamp amplifier (Axopatch 200, Molecular Devices) and using the pCLAMP10 software (Molecular Devices). Data were sampled at 2-5 kHz and filtered at $1 \mathrm{kHz}$, and were analyzed with Clampfit 10 (Molecular Devices) and SigmaPlot 9.0 (Systat Software Inc.). Data were calculated and plotted as the mean \pm standard error of the mean (SEM). All electrophysiological recordings were performed at $23^{\circ} \mathrm{C}$.

Sperm intracellular pH measurements. After sperm sample liquefaction (30 min at $37^{\circ} \mathrm{C}$ ), highly motile sperm were recovered by swim-up separation for $1 \mathrm{~h}$ in $\mathrm{HTF}$ (see above), at $37^{\circ} \mathrm{C}$ in an atmosphere of $5 \% \mathrm{CO}_{2}-95 \%$ air. Non-capacitated sperm were diluted to $10^{7}$ sperm/mL with modified HTF (mHTF: $4 \mathrm{mM} \mathrm{NaHCO}$ 
and $21 \mathrm{mM}$ HEPES were used to replace $25 \mathrm{mM} \mathrm{NaHCO}_{3}$ in $\mathrm{HTF}$ ) and loaded with BCECF $(2 \mu \mathrm{M}$, cell-permeant, dual-excitation ratiometric fluorescent $\mathrm{pH}$ probe $)$ in the dark at $37^{\circ} \mathrm{C}$ for $30 \mathrm{~min}$. Fluorescence intensity was measured with an Aminco Bowman II spectrofluorometer $\left(\lambda_{\mathrm{Ex} 1}=508, \lambda_{\mathrm{Ex} 2}=450, \lambda_{\mathrm{Em}}=535\right)$. Noncapacitated sperm were allowed to stabilize until there was no change, or a small continuous change, in the fluorescent signal. Then Alb or Fab were added and changes in fluorescence were recorded for $400 \mathrm{~s}$ at a frequency of $0.5 \mathrm{~Hz}$. High concentrations of Alb likely to cause quenching of fluorescent dyes, so $75 \mu \mathrm{M}$ was the highest concentration tested. For the $\mathrm{C} 6$ group, non-capacitated sperm were incubated with $20 \mu \mathrm{M} \mathrm{C} 6$ for $1 \mathrm{~h}$ before the experiment. Conversion of the BCECF Ratio $(508 / 450 \mathrm{~nm})$ to $\mathrm{pH}$ value was performed using a calibration curve adjusted with a conventional $\mathrm{pH}$ electrode as previous described ${ }^{28}$. Statistical analyses were performed using the Dunnett Test.

Sperm intracellular $\mathrm{Ca}^{2+}$ measurements. Non-capacitated sperm were diluted to $10^{7}$ sperm/mL with mHTF and loaded with Fluo3-AM $(2 \mu \mathrm{M}$, cell-permeant fluorescent $\mathrm{Ca}^{2+}$ probe) in the dark at $37^{\circ} \mathrm{C}$ for $30 \mathrm{~min}$. Non-capacitated sperm were allowed to stabilize before $75 \mu \mathrm{M}$ Alb was added and the changes in fluorescence intensity were measured $\left(\lambda_{\mathrm{Ex}}=505\right.$ and $\left.\lambda_{\mathrm{Em}}=525\right)$ for $950 \mathrm{~s}$ at a frequency of $0.5 \mathrm{~Hz}$. The increase in $\left[\mathrm{Ca}^{2+}\right]_{\mathrm{i}}$ was triggered by adding $15 \mu \mathrm{M}$ progesterone. Raw intensity values were normalized as $[(\mathrm{F} / \mathrm{F} 0)-1]$, where $\mathrm{F}$ is fluorescence intensity at time $t$ and $F 0$ is the mean of $F$ taken during the control period. In some studies, $10 \mu \mathrm{M}$ ionomycin was applied after progesterone to study the ionophore-mediated rise in $\left[\mathrm{Ca}^{2+}\right]_{\mathrm{i}}$ and demonstrate that Alb did not alter dye loading; in this case normalization was to ionomycin induced peak $\left[\mathrm{Ca}^{2+}\right]_{\mathrm{i}}$. Statistical analyses were performed using the Dunnett Test.

Sperm acrosome reaction measurements. Sperm suspensions were diluted to $10^{7} \mathrm{sperm} / \mathrm{mL}$ with HTF and incubated $3-4 \mathrm{~h}$ at $37^{\circ} \mathrm{C}$ in an atmosphere of $5 \%$ $\mathrm{CO}_{2}-95 \%$ air in presence of $5 \mathrm{mg} / \mathrm{mL}$ BSA to promote capacitation, or in absence of BSA for non-capacitating conditions. The effect of different concentrations of Alb, in the presence or absence of $20 \mu \mathrm{M}$ C6 were tested. Spermatozoa were incubated for $30 \mathrm{~min}$ at $37^{\circ} \mathrm{C}$ with $15 \mu \mathrm{M}$ progesterone, spotted on slides, air-dried and stained with FITC-coupled Pisum sativum agglutinin (FITC-PSA, $25 \mu \mathrm{g} / \mathrm{mL}$ in PBS) for $40 \mathrm{~min}$ at room temperature. The presence of an intact acrosome was assessed in at least 200 cells per condition using an upright Nikon Optiphot II microscope equipped with epifluorescence optics. Statistical analyses were performed using Dunnett Test.

Neutrophil ROS measurement. Human neutrophils were isolated from peripheral blood and re-suspended in Hank's Balanced Salt Solution (HBSS) comprising 138 $\mathrm{mM} \mathrm{NaCl}, 5.4 \mathrm{mM} \mathrm{KCl}, 0.34 \mathrm{mM} \mathrm{Na}_{2} \mathrm{HPO}_{4}, 0.44 \mathrm{mM} \mathrm{KH}_{2} \mathrm{PO}_{4}, 1.3 \mathrm{mM} \mathrm{CaCl}_{2}, 0.5$ $\mathrm{mM} \mathrm{MgCl}, 0.4 \mathrm{mM} \mathrm{MgSO}_{4}, 4.2 \mathrm{mM} \mathrm{NaHCO}_{3}, 5.5 \mathrm{mM}$ glucose, and $20 \mathrm{mM}$ HEPES, $\mathrm{pH}$ 7.2, and dispensed into white 96-Well Immuno Plates $\left(2 \times 10^{5}\right.$ cells/ well). Neutrophils were incubated with $500 \mu \mathrm{M}$ Luminol (Sigma) and different concentrations of Alb for $30 \mathrm{~min}$ at $37^{\circ} \mathrm{C}$. For the $\mathrm{C} 6$ group, neutrophils were preincubated with $20 \mu \mathrm{M}$ C6 for 30 min before the incubation with Luminol and Alb. After incubation, neutrophils were stimulated with fMLF and the chemiluminescence was measured immediately every $1 \mathrm{~min}$ for $60 \mathrm{~min}$ using Fluoroskan FL (ThermoFisher) equipped with internal software SkanIt 2.6.

Neutrophil elastase measurement. Human neutrophils were washed and resuspended in HBSS. Elastase release from neutrophils was evaluated using Elastase Substrate I, Colorimetric, AAPV-pNA (Millipore). All experiments were performed in polypropylene microcentrifuge tubes. Following the $30 \mathrm{~min}$ incubation with $\mathrm{Alb}$ or control protein $\mathrm{Fab}$ at $37^{\circ} \mathrm{C}$ neutrophils $\left(6.5 \times 10^{5}\right.$ cells per tube) were subjected to stimulation using $1 \mu \mathrm{M}$ fMLF. All cells were incubated for another $5 \mathrm{~min}$ and subsequently centrifuged at $400 \times g$ for $5 \mathrm{~min}$. The cell free supernatant was added to individual microplate wells to achieve a total reaction volume of $200 \mu \mathrm{L}$ per well and an AAPV-pNA concentration of $0.4 \mathrm{mM}$. Reactions were performed at $37^{\circ} \mathrm{C}$ for $1 \mathrm{~h}$, following which absorbance was measured at $405 \mathrm{~nm}$. An extinction coefficient of $8.8 \times 10^{3} \mathrm{~cm} / \mathrm{M}$ was used to calculate the units of elastase released. This number was then divided by the total neutrophil elastase content as determined from the Triton X-100 incubated neutrophils yielding the percentage of total elastase release for each group.

Two color smTIRF and photobleaching. HEK293T cells were seeded on glass bottom dishes (Chemglass Life Science) and transfected with hHv1-G199L-TFP. The surface density of channel molecules was kept less than 200 in a $10 \times 10 \mu \mathrm{m}$ field to minimize the overlapping of multiple channels within a diffraction-limited spot. TAMRA-Alb was added in $100 \mathrm{mM}$ HEPES, $90 \mathrm{mM} \mathrm{NaCl}, 10 \mathrm{mM} \mathrm{KCl}$, $0.5 \mathrm{mM} \mathrm{CaCl}_{2}, 1.2 \mathrm{mM} \mathrm{MgCl}_{2}$, and $10 \mathrm{mM}$ glucose, $\mathrm{pH} 7.5$ to the dishes and incubate $30 \mathrm{~min}$ for reaching binding equilibrium. Cells were extensively washed to remove nonspecifically bound TAMRA-Alb before recording. Single protein molecules or complexes at the surface of live HEK293T cells were identified using TIRF microscope as described ${ }^{5}$. The critical angle for TIRF was adjusted using a CellTIRF illuminator (Olympus) and a high numerical aperture apochromat objective (150x, $1.45 \mathrm{NA})$ mounted on an automated fluorescence microscope controlled by Metamorph 7 software (Molecular Devices). Metamorph was used to simultaneously illuminate both fluorophores at a critical angle such that only 100 $\mathrm{nm}$ deep was illuminated. TAMRA was excited with the $561 \mathrm{~nm}$ laser line and TFP was excited with a $445 \mathrm{~nm}$ laser line. Emitted light signals were split with a $520 \mathrm{~nm}$ dichroic mirror mounted in a DualView adapter (Photometrics), which allows each wavelength to be directed to one half of a back-illuminated EM-CCD. Stoichiometry was assessed by simultaneous photobleaching with continual excitation. Data were captured as movies of 100-370 frames acquired at $1 \mathrm{~Hz}$.

Data were analyzed as previously described ${ }^{5}$. When TAMRA was with TFP in the same cell, the data for each fluorophore were saved as separate stacks and processed in an identical manner. The Manders' coefficient of colocalization (MMC) between fluorophores was determined by unbiased intensity correlation analysis using the Coloc2 plugin in ImageJ (Windows version) to confirm overlap of the two molecules. Fluorescence measured from each region was plotted versus time to determine the number of bleaching steps at each point. Statistical analyses to calculate estimated confidence with which stoichiometry could be inferred from the observed data and $\theta$, the probability of successfully observing each possible photobleaching event, were performed in R Studio, based on methods developed by Hines $^{67}$. The densities of colocalized and single fluorescent spots were determined following thresholding and watershed separation in ImageJ. Then the particle number was counted in separate regions for 3-5 cells per group using the Analyze particles plugin.

Single-molecule photobleaching events are missed in practical application to biological systems because of fluorophore prebleaching, the quantum efficiency of the fluorophore, and the time resolution of smTIRF system; in our studies, the error is estimated to be less than $10 \%$ as previously demonstrated ${ }^{5,46}$.

Live cell FRET microscopy. Donor-decay time-course was studied as before ${ }^{45}$, using an Olympus inverted epi-fluorescence microscope. HEK293T cells were seeded on glass bottom dishes (Chemglass Life Science) and transfected with hHv1TFP and T-Alb-VFP variants. Cells were recorded in a solution comprising 100 mM HEPES, $90 \mathrm{mM} \mathrm{NaCl}, 10 \mathrm{mM} \mathrm{KCl}, 0.5 \mathrm{mM} \mathrm{CaCl}_{2}, 1.2 \mathrm{mM} \mathrm{MgCl}_{2}$, and $10 \mathrm{mM}$ glucose, $\mathrm{pH}$ 7.5. Resting membrane potential (RMP) was altered by isotonic replacement of extracellular $\mathrm{NaCl}$ with $\mathrm{KCl}$. For EGTA studies, $1 \mathrm{mM}$ was added to the $10 \mathrm{mM} \mathrm{KCl}$ bath solution. TFP was excited at $445 \mathrm{~nm}$ and the emission collected through a $470-500 \mathrm{~nm}$ bandpass filter, VFP was excited at $514 \mathrm{~nm}$ and the emission collected through a 525-575 nm filter. Images were captured using a scientific camera controlled by Metamorph 7 software (Molecular Devices) and were analyzed with Image $\jmath^{5,45}$.

Protein-peptide in silico docking. Unguided docking was performed using the amino acid sequence of the S3-S4 loop peptide of hHv1 (F190 to L200) and the crystal structure of Alb (PDB: 1BM0), to predict potential binding sites, using HPEPDOCK web server ${ }^{48}$.

Molecular dynamics simulations. The initial structure of the dimeric hHvl (only the transmembrane region: residues G90 to I218) at resting state was adopted from our previous study where the model was generated and refined by molecular dynamics (MD) simulations using spectroscopic constraints ${ }^{43}$. The hHvl modeling structure was embedded in an explicit POPC lipid bilayer using the program VMD $1.93^{68}$, while the crystal structure of Alb (PDB 1BM0; residues S5 to A582) was placed in the extracellular side with its DII domain and the two potential binding sites facing the S3-S4 loops of hHv1, fully solvated in a $100 \mathrm{mM} \mathrm{KCl}$ solution. The final system contained $\sim 185,000$ atoms and was electrically neutral. The H193 on the subunit $\mathrm{A}$ of $\mathrm{hHvl}$ was modeled in a protonated state to form hydrogen bonding interactions with E188 and E292 of Alb. Of note, the two glutamate residues could be the potential hydrogen bond donors as well.

The system was initially minimized for 5000 steps, followed by $10 \mathrm{~ns}$ equilibration with positional restraints $\left(0.5 \mathrm{kcal} / \mathrm{mol} / \AA^{2}\right)$ being applied to the backbone of the whole Alb and the transmembrane helices of hHv1 to relax with the lipids. Then, the positional restraints were removed except the intracellular half of the transmembrane helicesS4 of hHvl (residues R205 to I218), which were used to maintain the dimeric interface of hHvl as its cytosolic coiled-coil structure was excluded from the model to minimize the size of the system. The distance of center of mass between the side-chain heavy atoms of hHvl-H193 (subunit A) and AlbH288, and hHv1-H193 (subunit B) and Alb-H338 was gradually decreased (approaching to $5 \AA$ ) in 10 ns to pull Alb toward hHvl using the Colvars module ${ }^{69}$ Meanwhile, the S1-S2 loops of the dimeric hHv1 were given a distance restraint for optimized Alb contact with hHvl. After that, 150 ns simulations were performed with different combinations of distance restraints between hHv1-H193 and their potential partners on Alb (Supplementary Table 4) to explore the interacting networks at the two binding sites and other contact regions of the complex where spontaneously and transiently formed hydrogen bonds between $\mathrm{hHvl}$ and Alb had been found during the simulations. We used the distance restraints without angle restraints to determine if the enforced interaction was favorable. If the restrained interaction was favorable, a correct hydrogen-bonding angle formed automatically (Supplementary Fig. 14).

During the simulations, we found that E294 and E297 on Alb were close to I202 of hHv1, a helix turn upward the first gating charge R205, making E294 and E297 the probable negative countercharges activating the outward movement of the S4 
helices of the dimeric hHv1. Thus, we generated and simulated another system of the Alb and hHvl complex using the "up" state hHv1 (residues G90 to K221) from our previous study ${ }^{43}$. The simulation protocol was similar to the resting state model system with a total simulation time of $150 \mathrm{~ns}$.

The MD simulations were carried out in the periodic boundary conditions with a time step of $2 \mathrm{fs}$ using the NAMD 2.14 software program ${ }^{49}$. The CHARMM36 parameter set ${ }^{70}$ was used for proteins, lipids and ions, and the TIP3P model for water. The temperature and pressure were constrained at $300 \mathrm{~K}$ and $1 \mathrm{~atm}$, respectively, using the Langevin dynamics and the Nose-Hoover Langevin piston method $^{71}$. The long-range electrostatic force was calculated with the particle-mesh Ewald method ${ }^{72}$, and the short-range electrostatic and van der Waals interactions were smoothly switched off at $10-12 \AA$.

The evaluate the stability of the binding interactions of the two proteins, the two systems were further subjected to microsecond scale long simulations with ANTON $2^{50}$, a special-purpose computer for long time scale MD simulations (Supplementary Fig. 15, 16, 17). The simulations were performed with 16 pairs of distance restraints, as shown in Supplementary Table 4, (derived from the MD simulations with NAMD) for $1.0 \mu \mathrm{s}$. These pairwise interactions were selected based on the examination of our initial MD simulation results with NAMD and our functional data. After that, another $0.5 \mu$ s run was performed only with the distance restraints being replaced by positional restraints on the backbone atoms of binding site residues (site 1: H288, E188, E292, R160, E153, and site 2: Y334, F374, R337, H338) of Alb and the backbone and $\mathrm{C}_{\beta}, \mathrm{C}_{\gamma}$ atoms of hHv1-H193. In all these ANTON2 simulations, positional restraints were applied on the alpha carbon atoms of the intracellular part of the transmembrane helices of hHvl with the positional restraints on the backbone atoms of the intracellular part of S4 and the center-of-mass of the backbone atoms of the first two domains of Alb. The force filed, temperature, pressure, and time step were the same as those used in the MD simulations with NAMD. The temperature and pressure were constrained using the Nose-Hoover thermostat and the semi-isotropic MTK barostat $^{71}$. PCA analysis was performed using the PyEMMA and Prody packages.

Statistics. Statistical analyses were performed using the Dunnett Test, ${ }^{*} P<0.05$, ${ }^{* *} P<0.01,{ }^{* * *} P<0.001$. Data are presented, where indicated as the mean \pm standard error of the mean (SEM). The number of replicates for each study are described in the legends.

Reporting summary. Further information on research design is available in the Nature Research Reporting Summary linked to this article.

\section{Data availability}

The data that support this study are available form the corresponding author upon reasonable request. The crystal structure of Alb that was used for molecular dynamics simulation is from Protein Data Bank entry 1BM0. The EPR-derived structural model of hHvl and the computational model of the Alb-hHvl complex are available from the corresponding author upon request. Source data is provided with this paper. Source data are provided with this paper.

Received: 8 October 2020; Accepted: 2 June 2021;

Published online: 22 June 2021

\section{References}

1. Roos, A. \& Boron, W. F. Intracellular pH. Physiol. Rev. 61, 296-434 (1981).

2. Ramsey, I. S., Moran, M. M., Chong, J. A. \& Clapham, D. E. A voltage-gated proton-selective channel lacking the pore domain. Nature 440, 1213-1216 (2006).

3. Sasaki, M., Takagi, M. \& Okamura, Y. A voltage sensor-domain protein is a voltage-gated proton channel. Science 312, 589-592 (2006).

4. DeCoursey, T. E. Voltage-gated proton channels: molecular biology, physiology, and pathophysiology of the $\mathrm{H}(\mathrm{V})$ family. Physiol. Rev. 93, 599-652 (2013).

5. Zhao, R. et al. Role of human Hv1 channels in sperm capacitation and white blood cell respiratory burst established by a designed peptide inhibitor. Proc. Natl Acad. Sci. USA 115, E11847-E11856 (2018).

6. Kirichok, Y., Navarro, B. \& Clapham, D. E. Whole-cell patch-clamp measurements of spermatozoa reveal an alkaline-activated $\mathrm{Ca} 2+$ channel. Nature 439, 737-740 (2006).

7. Lishko, P. V., Botchkina, I. L., Fedorenko, A. \& Kirichok, Y. Acid extrusion from human spermatozoa is mediated by flagellar voltage-gated proton channel. Cell 140, 327-337 (2010).

8. Morgan, D. et al. Voltage-gated proton channels maintain $\mathrm{pH}$ in human neutrophils during phagocytosis. Proc. Natl Acad. Sci. USA 106, 18022-18027 (2009).
9. Ramsey, I. S., Ruchti, E., Kaczmarek, J. S. \& Clapham, D. E. Hv1 proton channels are required for high-level NADPH oxidase-dependent superoxide production during the phagocyte respiratory burst. Proc. Natl Acad. Sci. USA 106, 7642-7647 (2009).

10. El Chemaly, A. et al. VSOP/Hvl proton channels sustain calcium entry, neutrophil migration, and superoxide production by limiting cell depolarization and acidification. J. Exp. Med 207, 129-139 (2010).

11. Musset, B. et al. Aspartate 112 is the selectivity filter of the human voltagegated proton channel. Nature 480, 273-277 (2011).

12. Tombola, F., Ulbrich, M. H. \& Isacoff, E. Y. The voltage-gated proton channel Hv1 has two pores, each controlled by one voltage sensor. Neuron $\mathbf{5 8}, 546-556$ (2008).

13. Takeshita, K. et al. X-ray crystal structure of voltage-gated proton channel. Nat. Struct. Mol. Biol. 21, 352-357 (2014).

14. Ballmer, P. E. Causes and mechanisms of hypoalbuminaemia. Clin. Nutr. 20 271-273 (2001).

15. Quinlan, G. J., Martin, G. S. \& Evans, T. W. Albumin: biochemical properties and therapeutic potential. Hepatology 41, 1211-1219 (2005).

16. Sugio, S., Kashima, A., Mochizuki, S., Noda, M. \& Kobayashi, K. Crystal structure of human serum albumin at 2.5 A resolution. Protein Eng. 12, 439-446 (1999).

17. Elzanaty, S., Erenpreiss, J. \& Becker, C. Seminal plasma albumin: origin and relation to the male reproductive parameters. Andrologia 39, 60-65 (2007).

18. Casslen, B. \& Nilsson, B. Human uterine fluid, examined in undiluted samples for osmolarity and the concentrations of inorganic ions, albumin, glucose, and urea. Am. J. Obstet. Gynecol. 150, 877-881 (1984).

19. Laverge, H., De Sutter, P., Desmet, R., Van der Elst, J. \& Dhont, M. Prospective randomized study comparing human serum albumin with fetal cord serum as protein supplement in culture medium for in-vitro fertilization. Hum. Reprod. 12, 2263-2266 (1997).

20. Balaban, B., Sakkas, D. \& Gardner, D. K. Laboratory procedures for human in vitro fertilization. Semin Reprod. Med 32, 272-282 (2014).

21. Rabaglia, J. L., Gonzalez, R., Moore, E. E. \& Harken, A. H. Pooled human albumin primes neutrophils. J. Card. Surg. 17, 209-213 (2002).

22. Dias, I. H. et al. Activation of the neutrophil respiratory burst by plasma from periodontitis patients is mediated by pro-inflammatory cytokines. J. Clin. Periodontol. 38, 1-7 (2011)

23. Seredenina, T., Demaurex, N. \& Krause, K. H. Voltage-gated proton channels as novel drug targets: from NADPH oxidase regulation to sperm biology. Antioxid. Redox Signal 23, 490-513 (2015).

24. Fernandez, A., Pupo, A., Mena-Ulecia, K. \& Gonzalez, C. Pharmacological modulation of proton channel $\mathrm{Hvl}$ in cancer therapy: future perspectives. Mol. Pharm. 90, 385-402 (2016).

25. Austin, C. R. The capacitation of the mammalian sperm. Nature 170, 326 (1952).

26. Lishko, P. V. \& Kirichok, Y. The role of Hvl and CatSper channels in sperm activation. J. Physiol. 588, 4667-4672 (2010).

27. Puga Molina, L. C. et al. Molecular basis of human sperm capacitation. Front Cell Dev. Biol. 6, 72 (2018).

28. Chavez, J. C. et al. Acrosomal alkalization triggers $\mathrm{Ca}(2+)$ release and acrosome reaction in mammalian spermatozoa. J. Cell Physiol. 233, 4735-4747 (2018).

29. Lishko, P. V., Botchkina, I. L. \& Kirichok, Y. Progesterone activates the principal Ca2+ channel of human sperm. Nature 471, 387-391 (2011).

30. Darszon, A., Nishigaki, T., Beltran, C. \& Trevino, C. L. Calcium channels in the development, maturation, and function of spermatozoa. Physiol. Rev. 91, 1305-1355 (2011).

31. Xia, J. \& Ren, D. The BSA-induced Ca2+ influx during sperm capacitation is CATSPER channel-dependent. Reprod. Biol. Endocrinol. 7, 119 (2009).

32. Winterbourn, C. C., Kettle, A. J. \& Hampton, M. B. Reactive oxygen species and neutrophil function. Annu Rev. Biochem 85, 765-792 (2016).

33. DeCoursey, T. E., Morgan, D. \& Cherny, V. V. The voltage dependence of NADPH oxidase reveals why phagocytes need proton channels. Nature 422, 531-534 (2003).

34. DeCoursey, T. E. During the respiratory burst, do phagocytes need proton channels or potassium channels, or both? Sci. STKE 2004, pe21 (2004).

35. Bedouhene, S., Moulti-Mati, F., Hurtado-Nedelec, M., Dang, P. M. \& ElBenna, J. Luminol-amplified chemiluminescence detects mainly superoxide anion produced by human neutrophils. Am. J. Blood Res 7, 41-48 (2017).

36. Wittmann, S., Frohlich, D. \& Daniels, S. Characterization of the human fMLP receptor in neutrophils and in Xenopus oocytes. Br. J. Pharm. 135, 1375-1382 (2002).

37. Worthen, G. S., Avdi, N., Buhl, A. M., Suzuki, N. \& Johnson, G. L. FMLP activates Ras and Raf in human neutrophils. Potential role in activation of MAP kinase. J. Clin. Invest 94, 815-823 (1994).

38. Amulic, B., Cazalet, C., Hayes, G. L., Metzler, K. D. \& Zychlinsky, A. Neutrophil function: from mechanisms to disease. Annu Rev. Immunol. 30, 459-489 (2012). 
39. Hondares, E. et al. Enhanced activation of an amino-terminally truncated isoform of the voltage-gated proton channel HVCN1 enriched in malignant B cells. Proc. Natl Acad. Sci. USA 111, 18078-18083 (2014).

40. Berger, T. K. et al. Post-translational cleavage of Hv1 in human sperm tunes pH- and voltage-dependent gating. J. Physiol. 595, 1533-1546 (2017).

41. Kleijkers, S. H. et al. Influence of embryo culture medium (G5 and HTF) on pregnancy and perinatal outcome after IVF: a multicenter RCT. Hum. Reprod. 31, 2219-2230 (2016).

42. Chavez, J. C., Darszon, A., Trevino, C. L. \& Nishigaki, T. Quantitative Intracellular $\mathrm{pH}$ Determinations in Single Live Mammalian Spermatozoa Using the Ratiometric Dye SNARF-5F. Front Cell Dev. Biol. 7, 366 (2019).

43. Li, Q. et al. Resting state of the human proton channel dimer in a lipid bilayer. Proc. Natl Acad. Sci. USA 112, E5926-5935 (2015).

44. Auer, S. et al. Silencing neurotransmission with membrane-tethered toxins. Nat. Methods 7, 229-236 (2010).

45. Plant, L. D., Xiong, D., Romero, J., Dai, H. \& Goldstein, S. A. N. Hypoxia produces pro-arrhythmic late sodium current in cardiac myocytes by SUMOylation of NaV1.5 channels. Cell Rep. 30, 2225-2236 e2224 (2020).

46. Zhao, R., Dai, H., Mendelman, N., Chill, J. H. \& Goldstein, S. A. N. Tethered peptide neurotoxins display two blocking mechanisms in the $\mathrm{K}(+)$ channel pore as do their untethered analogs. Sci. Adv. 6, eaaz3439 (2020).

47. Andersen, J. T. et al. Structure-based mutagenesis reveals the albumin-binding site of the neonatal Fc receptor. Nat. Commun. 3, 610 (2012).

48. Zhou, P., Jin, B., Li, H. \& Huang, S. Y. HPEPDOCK: a web server for blind peptide-protein docking based on a hierarchical algorithm. Nucleic Acids Res 46. W443-W450 (2018).

49. Phillips, J. C. et al. Scalable molecular dynamics with NAMD. J. Comput Chem. 26, 1781-1802 (2005)

50. Shaw, D. E. et al. in Proceedings of the International Conference for High Performance Computing, Networking, Storage and Analysis 41-53 (IEEE Press, New Orleans, Louisana; 2014).

51. Sanchez-Carranza, O., Torres-Rodriguez, P., Darszon, A., Trevino, C. L. \& Lopez-Gonzalez, I. Pharmacology of hSlo3 channels and their contribution in the capacitation-associated hyperpolarization of human sperm. Biochem Biophys. Res Commun. 466, 554-559 (2015).

52. Macdonald, R. R. \& Lumley, I. B. Endocervical pH measured in vivo through the normal menstrual cycle. Obstet. Gynecol. 35, 202-206 (1970).

53. Nishigaki, T. et al. Intracellular $\mathrm{pH}$ in sperm physiology. Biochem Biophys. Res. Commun. 450, 1149-1158 (2014).

54. Jin, S. K. \& Yang, W. X. Factors and pathways involved in capacitation: how are they regulated? Oncotarget 8, 3600-3627 (2017).

55. Ferrier, J. M. Apparent bicarbonate uptake and possible plasmalemma proton efflux in chara corallina. Plant Physiol. 66, 1198-1199 (1980).

56. Demarco, I. A. et al. Involvement of a Na+/HCO-3 cotransporter in mouse sperm capacitation. J. Biol. Chem. 278, 7001-7009 (2003).

57. Lishko, P. V. et al. The control of male fertility by spermatozoan ion channels. Annu Rev. Physiol. 74, 453-475 (2012).

58. Decoursey, T. E. Voltage-gated proton channels and other proton transfer pathways. Physiol. Rev. 83, 475-579 (2003).

59. Demaurex, N. et al. Proton currents in human granulocytes: regulation by membrane potential and intracellular pH. J. Physiol. 466, 329-344 (1993).

60. Matthay, M. A. et al. Acute respiratory distress syndrome. Nat. Rev. Dis. Prim. 5, 18 (2019).

61. Kokot, K., Teschner, M., Schaefer, R. M. \& Heidland, A. Stimulation and inhibition of elastase release from human neutrophil-dependence on the calcium messenger system. Min. Electrolyte Metab. 13, 189-195 (1987)

62. Cestele, S. et al. Voltage sensor-trapping: enhanced activation of sodium channels by beta-scorpion toxin bound to the S3-S4 loop in domain II. Neuron 21, 919-931 (1998).

63. Geragotelis, A. D. et al. Voltage-dependent structural models of the human Hv1 proton channel from long-timescale molecular dynamics simulations. Proc. Natl Acad. Sci. USA 117, 13490-13498 (2020).

64. $\mathrm{Wu}, \mathrm{L}$. J. et al. The voltage-gated proton channel Hv1 enhances brain damage from ischemic stroke. Nat. Neurosci. 15, 565-573 (2012).

65. Jin, C. et al. HV1 acts as a sodium sensor and promotes superoxide production in medullary thick ascending limb of Dahl salt-sensitive rats. Hypertension 64, 541-550 (2014).

66. Musset, B. \& Decoursey, T. Biophysical properties of the voltage gated proton channel $\mathrm{H}(\mathrm{V}) 1$. Wiley interdisciplinary reviews. Membr. Transp. Signal. 1, 605-620 (2012).
67. Hines, K. E. Inferring subunit stoichiometry from single molecule photobleaching. J. Gen. Physiol. 141, 737-746 (2013).

68. Humphrey, W., Dalke, A. \& Schulten, K. VMD: visual molecular dynamics. J. Mol. Graph 14, 33-38 (1996). 27-38.

69. Fiorin, G., Klein, M. L. \& Hénin, J. Using collective variables to drive molecular dynamics simulations. Mol. Phys. 111, 3345-3362 (2013)

70. Klauda, J. B. et al. Update of the CHARMM all-atom additive force field for lipids: validation on six lipid types. J. Phys. Chem. B 114, 7830-7843 (2010).

71. Martyna, G. J., Tobias, D. J. \& Klein, M. L. Constant pressure molecular dynamics algorithms. J. Chem. Phys. 101, 4177-4189 (1994).

72. Essmann, U. et al. A smooth particle mesh Ewald method. J. Chem. Phys. 103 8577-8593 (1995)

73. Banh, R. et al. Hydrophobic gasket mutation produces gating pore currents in closed human voltage-gated proton channels. Proc. Natl Acad. Sci. USA 116, 18951-18961 (2019).

\section{Acknowledgements}

We are grateful for grant support from the NIH GM111716 (to S.A.N.G.); the US-Israe Binational Science Foundation (BSF 2013185 and 2017243) (to S.A.N.G); Universidad Nacional de Cuyo, Argentina (06/M116) (to L.S.M.); Agencia Nacional de Promoción Científica y Tecnológica, Argentina (PICT-2016-0894 and PICT-2018-4451) (to L.S.M.); Universidad Nacional de Cuyo, Argentina (06/J504) (to G.A.DB); and Agencia Nacional de Promoción Científica y Tecnológica, Argentina (PICT-2015-3681) (to G.A.DB) Universidad Nacional Autónoma de México, Dirección General de Asuntos del Personal Académico DGAP/PAPIIT IN200919 and Consejo Nacional de Ciencia y Tecnología, Fronteras 7 (to A.D.); NIH GM057846 (to E.P).

\section{Author contributions}

R.Z., R.S., L.S.M., A.D., and S.A.N.G. designed research; R.Z., H.D., R.J.A., G.A.D.B., G.O., R.S., M.A.P. performed research; and R.Z., H.D., G.A.D.B., G.O., R.S., E.P., L.S.M., A.D., and S.A.N.G. wrote the paper.

\section{Competing interests}

A patent application has been filed by S.A.N.G. based on part of this work. The authors declare no competing interests.

\section{Additional information}

Supplementary information The online version contains supplementary material available at https://doi.org/10.1038/s41467-021-24145-1.

Correspondence and requests for materials should be addressed to S.A.N.G

Peer review information Nature Communications thanks Vincenzo Carnevale, Thoma DeCoursey and Dagmar Wachten for their contribution to the peer review of this work

Reprints and permission information is available at http://www.nature.com/reprints

Publisher's note Springer Nature remains neutral with regard to jurisdictional claims in published maps and institutional affiliations.

Open Access This article is licensed under a Creative Commons Attribution 4.0 International License, which permits use, sharing, adaptation, distribution and reproduction in any medium or format, as long as you give appropriate credit to the original author(s) and the source, provide a link to the Creative Commons license, and indicate if changes were made. The images or other third party material in this article are included in the article's Creative Commons license, unles indicated otherwise in a credit line to the material. If material is not included in the article's Creative Commons license and your intended use is not permitted by statutory regulation or exceeds the permitted use, you will need to obtain permission directly from the copyright holder. To view a copy of this license, visit http://creativecommons.org/ licenses/by/4.0/.

(C) The Author(s) 2021 